\title{
A smog chamber comparison of a microfluidic derivatisation measurement of gas-phase glyoxal and methylglyoxal with other analytical techniques
}

\author{
X. Pang ${ }^{1,2}$, A. C. Lewis ${ }^{1,3}$, A. R. Rickard ${ }^{1,3}$, M. T. Baeza-Romero ${ }^{4}$, T. J. Adams ${ }^{5}$, S. M. Ball ${ }^{5}$, M. J. S. Daniels ${ }^{5}$, \\ I. C. A. Goodall ${ }^{5}$, P. S. Monks ${ }^{5}$, S. Peppe ${ }^{6}$, M. Ródenas García ${ }^{7}$, P. Sánchez ${ }^{7}$, and A. Muñoz ${ }^{7}$ \\ ${ }^{1}$ Wolfson Atmospheric Chemistry Laboratories, Department of Chemistry, University of York, Heslington, \\ York, YO10 5DD, UK \\ ${ }^{2}$ Key Laboratory of Wetland Ecology and Environment, Northeast Institute of Geography and Agroecology (NEIGAE), \\ Chinese Academy of Sciences, 4888 Shengbei Road, Changchun, 130102, China \\ ${ }^{3}$ National Centre for Atmospheric Science, University of York, Heslington, York, YO10 5DD, UK \\ ${ }^{4}$ Escuela de Ingeniería Industrial de Toledo, Universidad de Castilla la Mancha, Toledo, 45071, Spain \\ ${ }^{5}$ Department of Chemistry, University of Leicester, University Road, Leicester, LE1 7RH, UK \\ ${ }^{6}$ School of Earth and Environment, University of Leeds, Woodhouse Lane, Leeds, LS2 9JT, UK \\ ${ }^{7}$ Instituto Universitario Centro de Estudios Ambientales del Mediterráneo (CEAM-UMH), Spain
}

Correspondence to: A. C. Lewis (ally.lewis@york.ac.uk) or X. Pang (pangxbyuanj@gmail.com)

Received: 8 April 2013 - Published in Atmos. Meas. Tech. Discuss.: 24 June 2013

Revised: 11 December 2013 - Accepted: 19 December 2013 - Published: 3 February 2014

\begin{abstract}
A microfluidic lab-on-a-chip derivatisation technique has been developed to measure part per billion (ppbV) mixing ratios of gaseous glyoxal (GLY) and methylglyoxal (MGLY), and the method is compared with other techniques in a smog chamber experiment. The method uses $o$ (2, 3, 4, 5, 6-pentafluorobenzyl) hydroxylamine (PFBHA) as a derivatisation reagent and a microfabricated planar glass micro-reactor comprising an inlet, gas and fluid splitting and combining channels, mixing junctions, and a heated capillary reaction microchannel. The enhanced phase contact area-to-volume ratio and the high heat transfer rate in the micro-reactor resulted in a fast and highly efficient derivatisation reaction, generating an effluent stream ready for direct introduction to a gas chromatograph-mass spectrometer (GC-MS). A linear response for GLY was observed over a calibration range 0.7 to $400 \mathrm{ppbV}$, and for MGLY of 1.2 to $300 \mathrm{ppbV}$, when derivatised under optimal reaction conditions. The analytical performance shows good accuracy (6.6\% for GLY and $7.5 \%$ for MGLY), suitable precision $(<12.0 \%)$ with method detection limits (MDLs) of $75 \mathrm{pptV}$ for GLY and $185 \mathrm{pptV}$ for MGLY, with a time resolution of $30 \mathrm{~min}$. These MDLs are below or close to typical
\end{abstract}

concentrations of these compounds observed in ambient air. The feasibility of the technique was assessed by applying the methodology to quantify $\alpha$-dicarbonyls formed during the photo-oxidation of isoprene in the EUPHORE chamber. Good correlations were found between microfluidic measurements and Fourier Transform InfraRed spectroscopy (FTIR) with a correlation coefficient $\left(r^{2}\right)$ of 0.84 , Broadband Cavity Enhanced Absorption Spectroscopy (BBCEAS) $\left(r^{2}=0.75\right)$, solid phase micro extraction (SPME) $\left(r^{2}=\right.$ 0.89 ), and a photochemical chamber box modelling calculation $\left(r^{2}=0.79\right)$ for GLY measurements. For MGLY measurements, the microfluidic technique showed good agreement with BBCEAS $\left(r^{2}=0.87\right)$, SPME $\left(r^{2}=0.76\right)$, and the modeling simulation $\left(r^{2}=0.83\right)$, FTIR $\left(r^{2}=0.72\right)$ but displayed a discrepancy with Proton-Transfer Reaction Timeof-Flight Mass Spectrometry (PTR-ToF-MS) with $r^{2}$ value of 0.39 . 


\section{Introduction}

Glyoxal (GLY, $\mathrm{CH}(\mathrm{O}) \mathrm{CHO}$ ) and methylglyoxal (MGLY, $\left.\mathrm{CH}_{3} \mathrm{C}(\mathrm{O}) \mathrm{CHO}\right)$ are the most prevalent $\alpha$-dicarbonyls in the ambient atmosphere. They are found ubiquitously in urban, rural and remote ambient air owing to their wide variety of sources. Both $\alpha$-dicarbonyls are generated by the photochemical oxidation of anthropogenic and biogenic volatile organic compounds (VOCs) (Fu et al., 2008). They are important primary ring opening products in the $\mathrm{OH}$ initiated oxidation of monoaromatic compounds in the presence of $\mathrm{NO}_{\mathrm{x}}\left(\mathrm{NO}\right.$ and $\left.\mathrm{NO}_{2}\right)($ Calvert et al., 2002) and are photooxidation products from isoprene degradation as well as other biogenic VOCs (Calvert et al., 2000). GLY and MGLY have also attracted recent attention as potentially important contributors to global secondary organic aerosol (SOA) (Volkamer et al., 2009; Hallquist et al., 2009; Hoffmann et al., 1997), which can significantly impact climate, air quality and human health (Solomon et al., 2007; Mauderly and Chow, 2008). They are highly water-soluble and can form SOA through uptake into the aqueous phase of an aerosol particle or cloud droplet, followed by aqueous-phase reactions that lead to the formation of low-volatility organonitrogen/organosulphate/ oligomeric products (Hamilton et al., 2013; De Haan et al., 2009; Loeffler et al., 2006). GLY was reported to account for up to $15 \%$ of the mass of SOA in Mexico City (Volkamer et al., 2007).

However, observations of $\alpha$-dicarbonyls are rather limited, especially in rural and remote regions, where their mixing ratios are in the parts per trillion range, but can vary considerably depending on location. Low tens to hundreds of pptV mixing ratios (GLY: 15-1820 pptV and MGLY: 50-320 pptV) have been reported in rural, urban, and marine/open ocean environments (Lee et al., 1995; Volkamer et al., 2005a; Fu et al., 2008; Huisman et al., 2008; Sinreich et al., 2010; Vrekoussis et al., 2009; Munger et al., 1995; Spaulding et al., 2003; Washenfelder et al., 2011). Gaseous GLY mixing ratios have been observed up to $1.82 \mathrm{ppbV}$ in Mexico City (Volkamer et al., 2005a) and as high as $1.60 \mathrm{ppbV}$ above a Southeast Asia tropical rainforest (MacDonald et al., 2012). A global modelling study by Fu et al. (2008) shows that the majority of GLY (47\%) and MGLY $(79 \%)$ are produced by isoprene photo-oxidation, which is the most abundant VOC emitted into the atmosphere, with an estimated annual emission from the biosphere of $500 \mathrm{Tg} \mathrm{yr}^{-1}$ (Guenther et al., 1995). Other important sources of $\alpha$-dicarbonyls include the oxidation of unsaturated hydrocarbons like acetylene (GLY only) (Fu et al., 2008), alkenes (e.g. 2-methyl-3-buten-2-ol (Chan et al., 2009) and methyl substituted ethenes (Volkamer et al., 2007)) and monoaromatics (e.g. benzene (GLY only), toluene, xylene and 1,3,5-trimethylbenzene (MGLY only) (Volkamer et al., 2007, 2005b)). MGLY is also formed from the oxidation of acetone (Fu et al., 2008), hydroxyacetone and various oxygenated alkenes (e.g. methylacrolein and methylvinylketone
(Galloway et al., 2011)) with GLY formed from glycolaldehyde, acrolein and crotonaldehyde, which are important components of biomass burning plumes (Magneron et al., 2005). Modeling studies underestimate observations of total GLY column amounts measured from satellites, implying there is a substantial missing source of GLY, which accounts for about $50 \%$ of global GLY (Myriokefalitakis et al., 2008; Vrekoussis et al., 2009; Lerot et al., 2010).

GLY and MGLY are reactive and have lifetimes of the order of a few hours during the daytime. Their atmospheric sinks are controlled primarily by photolysis, reaction with $\mathrm{OH}$ and heterogeneous processes (Volkamer et al., 2005a, b; Fu et al., 2008). Therefore, both $\alpha$-dicarbonyls have been used as tracers of local vs. regional photochemistry of biogenic and anthropogenic VOCs, since their short lifetimes inhibit their long-range transport (Volkamer et al., 2007). Additionally, the photolysis of GLY and MGLY can be a significant source of tropospheric $\mathrm{HO}_{\mathrm{x}}\left(\mathrm{HO}+\mathrm{HO}_{2}\right)$ and their reactions with $\mathrm{OH}$ can contribute to the tropospheric burden of ozone (Fu et al., 2008). Since $\alpha$-dicarbonyls play important roles in the complex intermediary atmospheric chemistry of both ozone and SOA formations, they are attractive species to measure in order to test fundamental chemical understanding through mechanism evaluation of chemistry incorporated into predictive policy and scientific models such as the Master Chemical Mechanism (Saunders et al., 2003).

Despite the importance of $\alpha$-dicarbonyls in gas-phase atmospheric photochemistry and SOA formation, cheap and simple techniques for their rapid and sensitive in situ atmospheric measurement have not been readily available. In recent years several different optical/spectroscopic approaches have been proposed for atmospheric measurement of $\alpha$-dicarbonyls including Differential Optical Absorption Spectroscopy (DOAS) (Volkamer et al., 2005a; MacDonald et al., 2012), Broadband Cavity Enhanced Absorption Spectroscopy (BBCEAS) (Thalman and Volkamer, 2010; Washenfelder et al., 2008), Laser-Induced Phosphorescence (LIP) Spectrometry (Henry et al., 2012), and Fourier Transform InfraRed (FTIR) absorption spectroscopy (Connelly et al., 2012; Profeta et al., 2012). DOAS is a wellestablished technique used to identify trace gases by means of their individual differential (i.e. narrow band) absorption structures. Volkamer et al. demonstrated long-path differential optical absorption spectroscopy (LP-DOAS) in Mexico City to detect GLY with a detection limit of $150 \mathrm{pptV}$ using an atmospheric path length of $4420 \mathrm{~m}$ and integration times between 2 to 15 min (Volkamer et al., 2005a). GLY above a Southeast Asian tropical rainforest was measured by LP-DOAS and multi-axis (MAX) DOAS with a maximum mixing ratio of $1.6 \mathrm{ppbV}$ observed (MacDonald et al., 2012). Broadband methods based on making absorption measurements on gas samples within high finesse optical cavities are a promising, emerging detection technique for many atmospheric trace gases with broad, structured absorptions in the visible and ultraviolet spectral regions (Thalman and 
Volkamer, 2010; Washenfelder et al., 2008, 2011; Langridge et al., 2008). Cavity Enhanced Differential Optical Absorption Spectroscopy (CE-DOAS) has been shown to be capable of sensitive, in situ, single point measurements of GLY and MGLY with method detection limits (MDL calculated as 3 times standard deviation of the $\mathrm{S} / \mathrm{N}$ of the blank samples) as low as 43 and $255 \mathrm{pptV}$, respectively with one minute averaging (Thalman and Volkamer, 2010). A similar spectroscopic method, Broadband Cavity Enhanced Absorption Spectroscopy (BBCEAS) can achieve a MDL for GLY of $87 \mathrm{pptV}$ with one minute averaging (Washenfelder et al., 2008). A Laser-Induced Phosphorescence (LIP) technique has been employed for GLY measurement with a MDL of $18 \mathrm{pptV}$ in one minute and high time resolution (up to $3 \mathrm{~Hz}$ ) (Huisman et al., 2008, 2011). The LIP technique has recently been improved to enable the simultaneous measurement of GLY and MGLY with MDLs of $11 \mathrm{pptV}$ for GLY and $243 \mathrm{pptV}$ for MGLY in 5 min (Henry et al., 2012). However most of the optical methods reviewed above require some highly specialized, or high maintenance equipment, and require exchange of light sources, cavity mirrors and/or other optics in order to measure different species. More commonly available, FTIR is a recent-established method to measure gaseous GLY and MGLY at high concentrations (ppmV) (Profeta et al., 2011; Talukdar et al., 2011), however FTIR requires a path length of $100-200 \mathrm{~m}$ or longer to realise a low detection limits (ppbV). However, the application of FTIR to ambient GLY and MGLY measurements may be limited since some IR peak locations of GLY and MGLY can be likely obscured by adjacent $\mathrm{CO}_{2}, \mathrm{H}_{2} \mathrm{O}$ or $\mathrm{N}_{2} \mathrm{O}$ lines (Profeta et al., 2011).

Global GLY measurements have recently become available from satellites. Several space-borne imaging UV-VISnear IR spectrometers have been used to observe GLY from space and retrieve global total column GLY datasets, including the SCanning Imaging Absorption SpectroMeter for Atmospheric CartograpHY (SCIAMACHY), Global Ozone Monitoring Experiment (GOME) and GOME-2 instruments (Vrekoussis et al., 2010, 2009; Wittrock et al., 2006). Importantly, however, such satellites measurements require extensive validation by in situ measurements on the ground, ideally over a wide range of spatial and temporal scales. Satellite observations are good for providing a picture of the global distribution of a chemical species, but typically only produce one pixel of information covering a whole city at one time of day, insufficient to test the use of GLY and MGLY as tracers for fast photochemistry.

A common and versatile method for GLY and MGLY measurement uses the principle of chemical derivatisation with a derivatisation reagent, such as $o-(2,3,4,5,6$ pentafluorobenzyl)hydroxylamine (PFBHA) (Yu et al., 1995; Ho and Yu, 2002; Temime et al., 2007), 2,4dinitrophenylhydrazine (DNPH) (Ho and Yu, 2004; Lee et al., 1998) and pentafluorophenylhydrazine (PFPH) (Pang and Lewis, 2011; Ho and Yu, 2004), with subsequent analysis using bench-top GC-MS or High Performance Liquid Chromatography with ultraviolet detection (HPLC-UV). Such methods have acceptable sensitivity, good reproducibility and the considerable advantage of being able to quantify multiple compounds simultaneously (USA-EPA, 1999). For example GLY and MGLY were analysed by an on-sorbent PFBHA derivatisation technique with detection limits of 0.1 and $0.6 \mathrm{ppbV}$ and precisions of $2.2-5.3 \%$ and $0.7-6.4 \%$, respectively, after $4 \mathrm{~h}$ sampling time (Ho and Yu, 2002). MGLY has been measured in air by sampling with impingers filled with an aqueous PFBHA solution with a detection limit of $0.02 \mathrm{ppbV}$ after $3 \mathrm{~h}$ sampling time (Spaulding et al., 1999). Although derivatisation methods are proven for the measurement of GLY and MGLY, their drawbacks are long sampling times, with a lengthy and hands-on lab procedure of derivatisation and solvent extraction/evaporation following sampling of ambient air.

The techniques of Solid Phase Microextraction (SPME) using PFBHA on-fibre (Muñoz et al., 2011; Gomez Alvarez et al., 2012) and a mist chamber with PFBHA solution (Spaulding et al., 2002) can significantly reduce sampling times to 10 minutes with detection limits of $0.003 \mathrm{ppbV}$ for GLY and $0.01 \mathrm{ppbV}$ for MGLY, respectively. However, the preparation processes for PFBHA-coated fibres are complicated and labour intensive and the mist chamber derivatisation requires a large sample gas volume $(200 \mathrm{~L})$. Substantial benefits may be gained therefore from the employment of a methodology which can achieve a relatively short sampling time, eliminate the bench chemical processes for sample preparation, and be based on the universality of a GC-MS method. The key to making major improvements in GLY and MGLY measurements may therefore lie in near-automated sample preparation.

In this work, a simple and sensitive microfluidic derivatisation approach for GLY and MGLY analysis has been developed using accelerated and highly efficient derivatisation reactions between $\alpha$-dicarbonyls and a derivatisation reagent inside a micro-reactor. The micro-reactor provided a zone for gas sample mixing, chemical reaction and pre-concentration, essentially replacing three independent offline preparative steps. Hence we use the "lab-on-a-chip" moniker for the methodology. This methodology for GLY and MGLY builds on our previous reported techniques used to measure gas phase formaldehyde (Pang and Lewis, 2012). The microfluidic technique realises a near real-time analysis based on the rapid derivatisation reaction occurring simultaneously with air sampling at high chip temperatures, and without any postderivatisation treatment necessary on the samples. Combining the micro-reactor with the auto-sampler of a commercial GC-MS allows for continuous sampling and analysis with a time resolution of around $30 \mathrm{~min}$.

The performance of the microfluidic derivatisation technique is evaluated in this paper through simultaneous measurement of gaseous GLY and MGLY in various experiments performed at the European Photoreactor (EUPHORE), 
a highly instrumented, large-scale outdoor atmospheric simulation chamber located in Valencia, Spain. The microfluidic derivatisation technique was compared with FTIR, BBCEAS, PTR-ToF-MS, and SPME on observations of the temporal evolution of GLY and MGLY formed during the photo-oxidation of isoprene as well as with a detailed model simulation (the Master Chemical Mechanism (MCMv3.2)). The MCM model includes a series of "chamber specific" auxiliary mechanisms to account for EUPHORE chamber wall reactions (Bloss et al., 2005). The simulation MCM model can predict the evolutions of gaseous GLY and MGLY during the fast photochemical degradation of isoprene. Comparison of the MCM model results with observations made by the various measurement techniques enables one to consider whether the techniques have the requisite time resolution and sensitivity to follow the temporal changes in GLY and MGLY concentrations during chamber experiments.

\section{Experimental}

\subsection{Micro-reactor layout and analytical system}

The micro-reactor integrates three key functions: (1) a gas and liquid mixer and reactor, (2) reagent heating, and (3) sample pre-concentration. Gaseous mixtures containing GLY and MGLY were drawn into the micro-reactor through inlets by a clean air pump. The PFBHA derivatisation solution was then introduced into the micro-reactor simultaneously through a separate inlet by a peristaltic pump (Watson Marlow 205S, UK). The micro-reactor was fixed securely on the surface of a hotplate head for direct heating. GLY and MGLY in the gas stream reacted with PFBHA with high efficiency in the micro-channel through diffusion into the derivatisation solution which formed a laminar layer on the channel walls, analogous to a capillary chromatography stationary phase (Pang and Lewis, 2012). A stainless metal coil (1/16 inch in OD, 0.040 inch in ID and $50 \mathrm{~cm}$ in length) was connected to the outlet of the micro-reactor, and placed in an ice-salt bath with a temperature of $0^{\circ} \mathrm{C}$. The reaction solution eluting from the stainless coil was collected directly into a sample vial within the auto-sampler of the GC-MS. A more detailed description of the micro-reactor used is provided in a previous study (Pang et al., 2013).

\subsection{Materials and apparatus}

All chemicals (unless otherwise stated) were purchased from Sigma-Aldrich Company. Solvents including acetonitrile, methanol, ethanol, ethyl acetate, 2-propanol (HPLC grade) were purchased from Fisher (UK). The derivatisation reagent $o$-(2, 3, 4, 5, 6-pentafluorobenzyl) hydroxylamine (PFBHA) (99\%), was used directly in this study. The sample gases matrix including GLY and MGLY was pumped into the microreactor using a clean KNF diaphragm gas pump (PM20994022, Neuberger, Germany). The flow rate of sample gas was controlled over the range of $100-600 \mathrm{~mL} \mathrm{~min}^{-1}$ by a mass flow controller (Model GFC17, Aalborg, USA), itself calibrated by a gas flow meter (Alicat Scientific, USA). Flow of the derivatisation solution was generated and controlled by a peristaltic pump, varying from $20-120 \mu \mathrm{L} \mathrm{min}^{-1}$. The final volume of solution in the collection vial was around $0.5 \mathrm{~mL}$ under optimal experimental conditions. The optimisation of gas flow and derivatisation solution flow will be discussed in the Sect. 3.2 and the detailed information can be seen in Table 1.

A magnetic stirrer hotplate (RH Basic 2 IKAMAG, UK) with a contact thermometer was used to heat the microchip directly. Separation and detection of the PFBHA derivatives of GLY and MGLY were performed on a GC-MS system incorporating a Perkin Elmer (USA) Auto system XLGC and a Turbo Mass (USA) quardrupole MS equipped with a DB5 column $(60 \mathrm{~m} \times 0.25 \mathrm{~mm} \times 1.0 \mu \mathrm{m}$, length $\times$ internal diameter $\times$ film thickness). GC conditions were as follows: the GC oven temperature was initially set at $80^{\circ} \mathrm{C}$ for two minutes, programmatically ramped to $300^{\circ} \mathrm{C}$ with a temperature ramp of $8^{\circ} \mathrm{C} \mathrm{min}^{-1}$ and then held at $300^{\circ} \mathrm{C}$ for $1.5 \mathrm{~min}$. The solvent delay was set at $10 \mathrm{~min}$ to avoid possible damage to the MS detector. The GC injection mode was set as splitless. The temperatures of the GC inlet and GC-MS transfer line were kept at $250^{\circ} \mathrm{C}$. The mass spectrometer was operated in scan mode with a mass range of 100-500 Da to identify the most abundant ions. The selected ion chromatograms of the most abundant ion $\left(\left[\mathrm{C}_{6} \mathrm{~F}_{5} \mathrm{CH}_{2}\right]^{+}\right)$with $m / z=181 \mathrm{Da}$, which is a fragment of the PFBHA molecule, were used to quantify the concentration of derivatives in solution.

\subsection{Description of EUPHORE chamber and instrumentation}

All experiments were performed in the European PhotoReactor (EUPHORE), located in Valencia, Spain during the period of 28 June to 2 August 2012. EUPHORE consists of two FEP (Teflon) hemispherical chambers mounted on aluminium floor panels. The FEP foil is highly transparent even to short-wavelength solar radiation. The chamber used in the present study (Chamber A) has a volume of ca. $200 \mathrm{~m}^{3}$ and is protected by retractable hemispherical shutters when its exposure to sunlight is not required. The chamber is operated at ambient temperature and approximately atmospheric pressure using purified air, while two large fans provide homogeneous mixing of the chamber contents.

A FTIR spectrometer (NICOLET 550, MCT/B-detector) is coupled to a multi-pass White-Cell mirror system for long path in situ monitoring of gaseous reactants and products in the IR spectral range $\left(400-4000 \mathrm{~cm}^{-1}\right)$. The gold-coated mirrors of the cell allow for a total optical path length of $616 \mathrm{~m}$. Spectra are collected with $1 \mathrm{~cm}^{-1}$ resolution by averaging 280 scans over a 5 min period. Concentrations of GLY and MGLY in the chamber during the experiments were determined with improved analysis software developed 
Table 1. Optimal experimental conditions for the microfluidic derivatisation reaction between PFBHA and gaseous $\alpha$-dicarbonyls in the micro-reactor.

\begin{tabular}{lrrrrr}
\hline $\begin{array}{l}\text { PFBHA } \\
\text { concentration }\end{array}$ & $\begin{array}{r}\text { Temperature } \\
\left({ }^{\circ} \mathrm{C}\right)\end{array}$ & $\begin{array}{r}\text { Flow rate of d } \\
\text { dicarbonyls } \\
\left(\mathrm{mL} \mathrm{min}^{-1}\right)\end{array}$ & $\begin{array}{r}\text { Flow rate of } \\
\text { solution } \\
\left(\mu \mathrm{min}^{-1}\right)\end{array}$ & $\begin{array}{r}\text { Sampling time } \\
(\mathrm{min})\end{array}$ & $\begin{array}{r}\text { Volume of liquid } \\
\text { from outlet } \\
(\mathrm{mL})\end{array}$ \\
\hline $5 \times 10^{-4} \mathrm{~mol} \mathrm{~L}^{-1}$ & 60 & 200 & 40 & 30 & 0.5 \\
\hline
\end{tabular}

at CEAM and using calibrated reference spectra collected a priori (Ródenas, 2008). GLY and MGLY were monitored in the aldehydic C-H band, within the spectral region of 2750$2900 \mathrm{~cm}^{-1}$ for GLY and $2750-2920 \mathrm{~cm}^{-1}$ for MGLY. For the calibration of gases measured by FTIR, GLY was injected into the chamber with a syringe in repeated trials while MGLY was cross-calibrated against DOAS. The accuracies are estimated to be $8 \%$ and $15 \%$ for GLY and MGLY measurements by FTIR.

A custom-built Broadband Cavity Enhanced Absorption Spectroscopy (BBCEAS) instrument was also employed to monitor GLY and MGLY. The BBCEAS instrument has been developed for highly sensitive and target-selective in situ trace gas measurements such as atmospheric $\mathrm{NO}_{2}$ and $\mathrm{NO}_{3}$ (Langridge et al., 2008). A blue light emitting diode (LED) was employed as the light source, enabling simultaneous measurements of GLY, MGLY and $\mathrm{NO}_{2}$ based on their molecules' distinctive absorption spectral signatures in the wavelength range $430-486 \mathrm{~nm}$. The accuracy of the BBCEAS measurements is determined by two main considerations: (i) uncertainties in the reference absorption cross sections used to fit the differential spectral structure in the BBCEAS spectra to retrieve the target absorber concentrations, and (ii) uncertainties in determining the reflectivity of the cavity mirrors (i.e. the effective path length of the BBCEAS absorption measurement) and the proportion of the cavity occupied by the gas sample (the cavity mirrors are purged with synthetic air to prevent contamination). The overall accuracies of the BBCEAS measurements are estimated to be $7 \%$ for GLY and $10 \%$ for MGLY.

A PTR-ToF-MS instrument (Series I, Kore, UK) was also used to detect the gaseous MGLY. The PTR-ToF-MS technique is based on the chemical ionization of trace VOCs present in atmospheric samples by proton transfer reactions with the hydronium reagent ion $\left(\mathrm{H}_{3} \mathrm{O}^{+}\right)$. The product is a protonated molecular ion (VOC-H) ${ }^{+}$for each VOC which is then selected and quantified by time-of-flight mass spectrometry (Blake et al., 2009). The PTR-ToF-MS method is also applicable to oxygenated VOCs such as GLY and MGLY. However, one drawback to PTR-MS, common to on-line mass spectrometric techniques, is isobaric interferences between VOC species being sampled at the same $m / z$ value. GLY is isobaric with propanal and acetone and as a result GLY cannot be exactly quantified by PTR-ToF-MS in this study. Only MGLY was measured based on the $m / z=73 \mathrm{Da}$ signal for MGLY-H ${ }^{+}$.

A Solid Phase Microextraction (SPME) technique was also used to determine GLY and MGLY through PFBHA on-fibre derivatisation. Detailed descriptions of this methodology can be found in the previously published literature (Muñoz et al., 2011; Gomez Alvarez et al., 2012). Briefly, the fibres were conditioned in the injector of a GC at $250^{\circ} \mathrm{C}$, and loaded with PFBHA derivatisation reagent through the headspace of a $4 \mathrm{~mL}$ opaque amber vial containing a $17 \mathrm{mg} \mathrm{mL}^{-1}$ solution of PFBHA in water. Gas samples were extracted from the EUPHORE chamber by inserting the SPME holder into a built-in adapter in one of the chamber's flanges. Sampling time was $5 \mathrm{~min}$. GLY and MGLY were then quantified using a $6890 \mathrm{HP}$ GC with a flame ionization detector (GC-FID) through determination of their corresponding PFBHA derivatives on the SPME fibre.

The linear ranges, MDLs, sampling times and measuring times of the above GLY and MGLY measurement techniques are listed in Table 2.

\subsection{Preparing gaseous GLY and MGLY}

Pure GLY monomer was prepared from the solid trimerdihydrate (95\%) using a similar method as described in the literature (Volkamer et al., 2005b). MGLY is available commercially as $40 \%$ aqueous solutions wherein it exists primarily in its monohydrate and dihydrate forms. To obtain the unpolymerized and water-free form, the procedure described in Gurnick et al. (1981) was conducted with minor modifications. Flasks containing pure and unpolymerized GLY or MGLY were stored in liquid nitrogen prior to experimental use.

Pure GLY or MGLY was introduced into the EUPHORE chamber by passing a small flow of nitrogen through a coldtrap whilst allowing the trap to warm gently. After each addition, the chamber's contents were homogenously mixed by the fans and stabilized for five minutes thereby obtaining a stable GLY and MGLY gaseous mixture inside the chamber. Chamber measurements were taken for a period of $30 \mathrm{~min}$ before fast dilution down to a lower concentration by adding a large flow of clean air, allowing the chamber contents to mix thoroughly and measuring for a period of 30 min repeatedly until GLY and MGLY concentrations were below the detection limits. The detailed temporal variations of $\alpha$-dicarbonyl concentrations are shown in Fig. 1a. 
Table 2. Comparison between the microfluidic derivatisation technique and other methods including linear range and method detection limit (MDL is calculated on 3 times standard deviation of the $\mathrm{S} / \mathrm{N}$ of the blank samples).

\begin{tabular}{|c|c|c|c|c|c|c|c|}
\hline $\begin{array}{l}\text { Analytical } \\
\text { technique }\end{array}$ & $\begin{array}{r}\text { GLY } \\
\text { Linear range } \\
(\mathrm{ppbV})\end{array}$ & $\begin{array}{l}\mathrm{MDL}^{\mathrm{a}} \\
(\mathrm{ppbV})\end{array}$ & $\begin{array}{l}\text { MGLY } \\
\text { Linear range } \\
(\mathrm{ppbV})\end{array}$ & $\begin{array}{r}\text { MDL } \\
(\mathrm{ppbV})\end{array}$ & Measurement time & Sampling time & Ref. \\
\hline Microfluidic & $0.7-400$ & 0.08 & $1.2-300$ & 0.2 & $30 \mathrm{~min}$ & $30 \min ^{\mathrm{c}}$ & This study \\
\hline FTIR & $2.5-3500$ & 2.5 & $2.7-4200$ & 2.7 & $5 \mathrm{~min}$ & $5 \min ^{\mathrm{d}}$ & This study \\
\hline SPME & $1-130$ & 0.1 & $1-80$ & 0.15 & $25 \mathrm{~min}$ & $5 \min ^{\mathrm{c}}$ & This study \\
\hline PTR-ToF-MS & $-\mathrm{b}$ & - & $1.7-172$ & 1.5 & $5 \mathrm{~min}$ & $5 \min ^{\mathrm{d}}$ & This study \\
\hline BBCEAS & $0.05-300$ & 0.07 & $0.4-400$ & 1.0 & $20 s$ & $20 s^{d}$ & This study \\
\hline Mad LIP & - & 0.02 & - & - & $1 \mathrm{~min}$ & $1 \min ^{\mathrm{d}}$ & Huisman et al. (2008) \\
\hline LIP & - & 0.01 & - & 0.24 & $5 \mathrm{~min}$ & $5 \min ^{\mathrm{d}}$ & Henry et al. (2012) \\
\hline IBBCEAS & - & 0.09 & - & - & $1 \mathrm{~min}$ & $1 \min ^{\mathrm{d}}$ & Washenfelder et al. (2008) \\
\hline LED-CE-DOAS & - & 0.03 & - & 0.255 & $1 \mathrm{~min}$ & $1 \min ^{\mathrm{d}}$ & Thalman and Volkamer (2010) \\
\hline DOAS & - & 0.15 & - & - & $2-15 \min$ & $2-15 \min ^{\mathrm{d}}$ & Volkamer et al. (2005a) \\
\hline PFBHA-mist chamber & - & 0.003 & - & 0.01 & $30 \mathrm{~min}$ & $15 \min ^{\mathrm{c}}$ & Spaulding et al. (2002) \\
\hline PFBHA impinger & - & - & - & 0.02 & $30 \mathrm{~min}$ & $3 h^{c}$ & Spaulding et al. (1999) \\
\hline
\end{tabular}

${ }^{a}$ MDLs are 3 times standard deviation of the S/N of the blank samples. ${ }^{\mathrm{b}} \mathrm{NO}$ data available. ${ }^{\mathrm{c}}$ Offline techniques: sampling and measurement processes are separated.

d Online techniques: sampling and measurement processes occurring simultaneously.

The downward steps provide a series of gas phase calibrations of GLY and MGLY over a representative range of concentrations generated inside the EUPHORE chamber during experiments performed to investigate the photo-chemical oxidation of VOCs. The different chamber amounts of GLY and MGLY were used to test the instrument response and linearity, and assigned absolute mixing ratio values using the comeasurements by FTIR (ranging from $2.5 \mathrm{ppbV}$ to $405 \mathrm{ppbV}$ for GLY and from $5.4 \mathrm{ppbV}$ to $227 \mathrm{ppbV}$ for MGLY) and BBCEAS (ranging upwards from $1.0 \mathrm{ppbV}$ for GLY and $0.7 \mathrm{ppbV}$ for MGLY). Given their similar performances in the GLY and MGLY measurement experiments (Fig. $1 \mathrm{~b}$ and c), these two techniques were chosen as the reference instruments to evaluate the performance of the microfluidic derivatisation technique.

\subsection{Preparing standard solutions of PFBHA-dicarbonyl derivatives}

A set of known concentrations of the PFBHA-dicarbonyl derivatives were prepared in acetonitrile by mixing the $\alpha$ dicarbonyls with a PFBHA solution whose concentration is five times higher than the highest concentration of $\alpha$ dicarbonyls. Six standards of individual $\alpha$-dicarbonyls were prepared ranging from 5.6 to $33.6 \mu \mathrm{mol} \mathrm{L}^{-1}(5.6,11.2,16.8$, $22.4,28.0$ and $33.6 \mu \mathrm{mol} \mathrm{L}^{-1}$ ). They are equal to the quantities of $\alpha$-dicarbonyl derivatives formed by 6.7, 13.4, 20.1, $26.8,33.5$, and $40.2 \mathrm{ppbV}$ of gaseous $\alpha$-dicarbonyls under the chamber conditions $\left(30^{\circ} \mathrm{C}\right.$ at $\left.1 \mathrm{~atm}\right)$ reacting with PFBHA, assuming $100 \%$ reaction efficiencies under the optimal micro-reactor conditions shown in Table 1 , with a solution enriching ratio of 2.77 (Pang and Lewis, 2012). Given that stable gaseous standards for these compounds are unavailable, the liquid based calibration method would be a feasible approach for future field measurement by
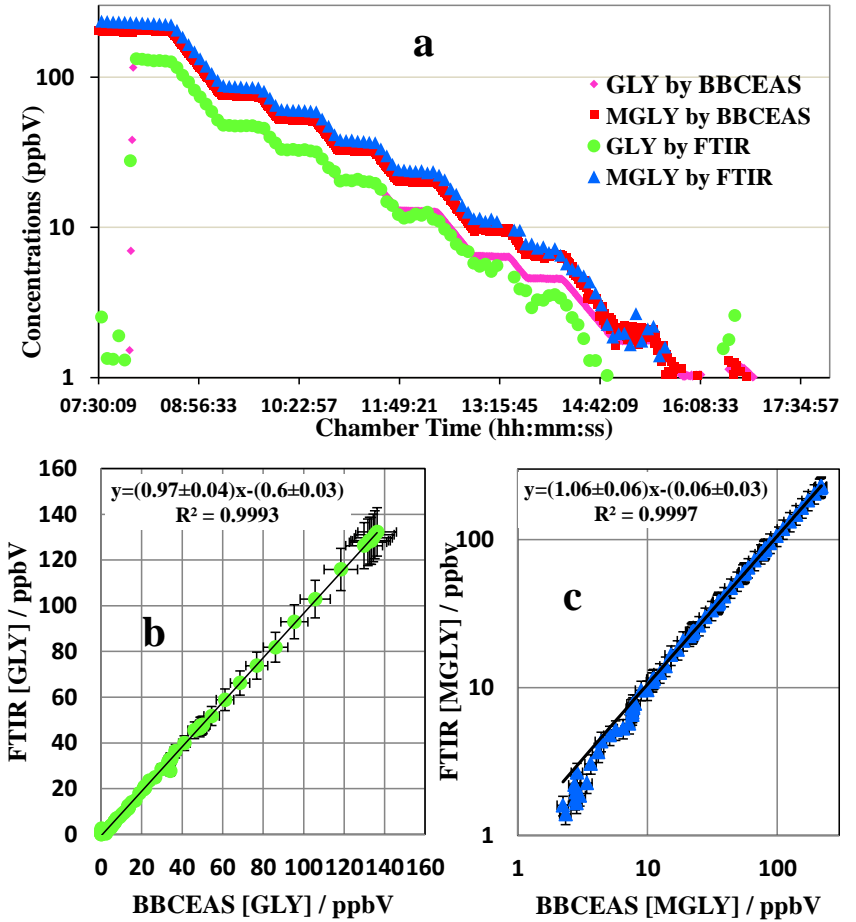

Fig. 1. Comparison between FTIR and BBCEAS measurements of GLY and MGLY. (a): Comparison between FTIR and BBCEAS measurements during the preparation of standard gaseous GLY and MGLY mixture in the EUPHORE chamber; (b): linear relationship between FTIR and BBCEAS for GLY measurement (error bars indicate the accuracies of FTIR and BBCEAS on GLY measurement); (c): linear relationship between FTIR and BBCEAS for MGLY measurement (error bars indicate the accuracies of FTIR and BBCEAS on MGLY measurement). The uncertainties of the slopes are less than $5 \%$. 
microfluidic technique, however, a separate methodology for in situ checks for inlet line losses and variations in collection efficiency still need to be devised.

\subsection{Accuracy and precision of the microfluidic derivatisation technique}

Standard $\alpha$-dicarbonyl mixtures of gases of three different concentrations were prepared in the chamber and used to check the method accuracy and precision. The accuracy of the method was evaluated by the relative error which is expressed as [(observed concentration - absolute concentration)/absolute concentration] $\times 100 \%$. Five consecutive samples for each concentration were collected. The absolute concentrations of GLY and MGLY are provided by the BBCEAS instrument chosen for its superior accuracy for these species. The concentrations were also calculated via the microfluidic derivatisation technique under the experimental conditions as shown in Table 1, using a peak height calculation based on the liquid calibration curves. Ignoring the uncertainties introduced by the FTIR and BBCEAS reference measurements themselves, the microfluidic derivatisation has two main sources of error: (i) the experimental uncertainties of microfluidic derivatisation technique, assessed using repeat samples under the identical experimental conditions, (ii) the instrumental uncertainties of the GC-MS response, assessed here by multiple injections of the same sample under the same instrumental conditions. The precision of the microfluidic derivatisation technique was determined by the replicate analyses $(n=5)$ of standard $\alpha$-dicarbonyl gases at three different concentrations under the same experimental conditions. The precision of the GC-MS instrument was calculated by repeated measurements $(n=5)$ for one sample under the same GC-MS instrumental conditions. The precision was expressed as the relative standard deviation $(\mathrm{RSD})=[($ standard deviation of observed concentration)/ (average of observed concentration)] $\times 100 \%$.

\subsection{EUPHORE isoprene photo-oxidation experiment}

The microfluidic derivatisation technique was employed through the whole summer 2012 "Pho-SOA" campaign in order to determine the gaseous GLY and MGLY concentrations in the EUPHORE chamber. To illustrate the microfluidic derivatisation technique capability, its performance on 25 July 2012 is chosen. For this particular experiment, isoprene photo-oxidation was carried out under relatively low $\mathrm{NO}_{\mathrm{x}}$ conditions (initial $\mathrm{VOC} / \mathrm{NO}_{\mathrm{X}}$ ratio of about 7). $226 \mathrm{ppbV}$ of isoprene was prepared by injecting $170 \mu \mathrm{L}$ of isoprene (liquid phase) into the chamber by a heated air flow. Sulfur hexafluoride $\left(\mathrm{SF}_{6}\right)$ was also added in order to monitor the chamber dilution (using FTIR). HONO was then continuously added into the chamber throughout the experiment so that a steady state of 5-8 ppbV of HONO was achieved (monitored by a Long Path Absorption Photometer (LOPAP) and FTIR) (Heland et al., 2001). This experiment was designed so that GLY and MGLY formation could be monitored throughout the entire chemical evolution of the isoprene photo-oxidation system. After the gases in the chamber were mixed by the fans and stabilized for $5 \mathrm{~min}$, the retractable half-spherical housing was opened exposing the chamber to solar radiation, photolysing HONO to produce $\mathrm{OH}$ radicals and thus initiating the photoxidation. The chamber was closed when the formation of ozone had reached a maximum (after about $400 \mathrm{~min}$ ). A comprehensive suite of instrumentation measuring gas and aerosol phase composition was available including those capable of sensitively monitoring the formation of GLY and MGLY (i.e. FTIR, BBCEAS, PTR-ToF-MS, and SPME). Each sample was collected over $30 \mathrm{~min}$ at $200 \mathrm{~mL} \mathrm{~min}^{-1}$ from the chamber giving a total volume of $6 \mathrm{~L}$ passed through the microfluidic derivatisation device. During the chamber experiment the next sample was collected whilst the first sample was measured on the GC-MS, and so on.

The composition and evolution of the gas phase components of the isoprene photo-oxidation chamber system was also simulated using a chamber optimised photochemical box model incorporating the comprehensive isoprene photooxidation scheme from the Master Chemical Mechanism (MCMv3.2) (Saunders et al., 2003). Detailed modelling of the chamber system is needed in order to design the experimental conditions and to understand the sources and sinks of the target species of interest. They allow comparisons between modelled and measured concentration profiles of not only ozone, $\mathrm{NO}_{\mathrm{x}}$ and the VOC of interest, but also a wide range of products and intermediates, such as GLY and MGLY, the temporal and chemical evolution of which provided invaluable information on the fast photo chemistry taking place - enabling us to test our chemical knowledge and understanding of the system under investigation.

The isoprene mechanism employed (along with an appropriate inorganic reaction scheme) contains 456 species and 1476 reactions, and was extracted directly from the MCM website: $\mathrm{http} / / \mathrm{mcm}$.leeds.ac.uk/MCM. The box model used in this study also includes a series of "chamber specific" auxiliary reactions adapted from previous work (Bloss et al., 2005) in order to take into account the typical background reactivity of the EUPHORE chamber walls as well as chamber dilution. The model was initiated with measured concentrations of isoprene, $\mathrm{NO}, \mathrm{NO}_{2}$ and ozone and constrained to measured HONO, temperature, and relative humidity. The photolysis rate parameterisations used in MCMv3.2 (Saunders et al., 2003) have been adjusted to replicate the photolytic conditions inside the EUPHORE chamber using measured values of $j\left(\mathrm{NO}_{2}\right)$. More details on the chamber modelling approach used can be found in Rickard et al. (2010). 


\section{Results and discussions}

The solvent, PFBHA concentration, microchip temperature, flow rates of gas and solution and reaction time can all affect the reaction efficiency of the derivatisation reaction and, therefore, an optimisation of the parameters is required to achieve the highest efficiency of derivatisation from each micro-preparative system. At the same time, in order to achieve a good separation, chromatographic conditions were optimised to the values described in Sect. 2.2. We have described previously the optimisation process and parameters for formaldehyde measurement (Pang and Lewis, 2012) and have undertaken a similar optimisation here including solvent selection, optimisations of PFBHA concentration, temperature, storage time, gas and solution flows. The detailed description of the solvent selection can be seen in our previous study (Pang et al., 2013).

The reaction between GLY/MGLY and PFBHA to form the corresponding pentafluorobenzyl oximes is a two-step process, as illustrated in Fig. 2. In the first step of the reaction, the PFBHA reacts with the $\alpha$-dicarbonyl through nucleophilic attack to form an amino alcohol. The second step is dehydration of the amino alcohol, which leads to formation of an oxime (Spaulding et al., 2002; Ho and Yu, 2002). When GLY and MGLY which contain two carbonyl groups are derivatised, three isomers (ZZ, EZ, EE isomers) are formed for GLY and four isomers (ZE, ZZ, EZ, EE isomers) are potentially formed for MGLY. However in practice, only two of the four MGLY isomers are formed in significant concentrations. The retention times of one GLY derivative and one of the observed MGLY derivatives are so close that their peaks overlap and therefore cannot be resolved sufficiently using the current instrument (Pang et al., 2013). Therefore, the GLY derivative with a retention time of $26.4 \mathrm{~min}$ and the MGLY derivative with retention time of 27.0 min were used to quantity analysis in this study (Pang et al., 2013).

\subsection{Optimisations of PFBHA concentration, reaction temperature and storage time}

Increasing the liquid phase PFBHA concentration from $1.0 \times$ $10^{-4}$ to $6 \times 10^{-4} \mathrm{~mol} \mathrm{~L}^{-1}$ (whilst the GLY and MGLY concentrations inside the EUPHORE chamber were held constant at $50 \mathrm{ppbV}$ ) was found to improve the $\alpha$-dicarbonylPFBHA derivatisation yield. Positive relationships existed between the $\alpha$-dicarbonyl derivative-oxime response and the PFBHA concentration for both GLY and MGLY under the experimental conditions with $200 \mathrm{~mL} \mathrm{~min}^{-1}$ flow rate of the sample gas, $40 \mu \mathrm{L} \mathrm{min}^{-1}$ derivatisation solution flow and the temperature of micro-reactor at $60{ }^{\circ} \mathrm{C}$ (Fig. 3a). In a $1.0 \times 10^{-4} \mathrm{~mol} \mathrm{~L}^{-1}$ PFBHA reaction solution, the signal responses (peak heights) of $\alpha$-dicarbonyl derivatives are equal to $25 \%$ of those of $\alpha$-dicarbonyl derivatives in $6.0 \times 10^{-4} \mathrm{~mol} \mathrm{~L}^{-1}$ PFBHA reaction solution. Since $6.0 \times 10^{-4} \mathrm{~mol} \mathrm{~L}^{-1}$ is close to the saturated solubility of

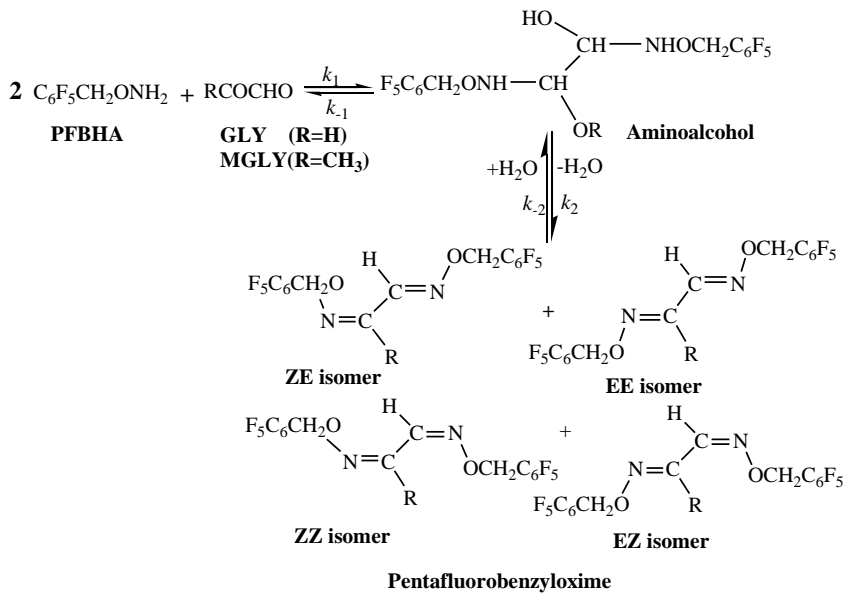

Fig. 2. Two-step process of derivatisation of GLY or MGLY with PFBHA to form the corresponding derivative oxime isomers. Three isomers are formed for GLY (ZE, EE, ZZ) and four isomers (ZE, $\mathrm{ZZ}, \mathrm{EZ}, \mathrm{EE}$ isomers) are potentially formed for MGLY.

PFBHA in acetonitrile, this was the maximum PFBHA concentration tested. According to the derivatisation mechanism there is a reaction equilibrium between PFBHA and the $\alpha$ dicarbonyl (R1) with the formation of an oxime derivative (PFBHA oxime):

2PFBHA + Dicarbonyl $\stackrel{K}{\Longleftrightarrow}$ PFBHA oxime

The equilibrium constant is expressed as Eq. (1)

$K=\frac{[\text { PFBHA oxime }]}{[\text { Dicarbonyl }] \cdot[\text { PFBHA }]^{2}}$.

Equation (1) can be rearranged into the following Eq. (2):

$[$ PFBHA oxime $]=K[\text { PFBHA }]^{2}[$ dicarbonyl $]$.

According to Eq. (2) more PFBHA oxime will be produced when using a higher concentration of PFBHA reagent, which forces the equilibrium of Reaction (1) heavily in favour of the oxime formation.

To the best of our knowledge, the influence of temperature on derivative yields has not previously been studied for $\alpha$-dicarbonyls. In this study the relative derivative signal responses of GLY and MGLY were found to increase from about $40 \%$ at $20^{\circ} \mathrm{C}$ to $96 \%$ at $50{ }^{\circ} \mathrm{C}$ as illustrated in Fig. $3 \mathrm{~b}$ with experimental conditions of $200 \mathrm{~mL} \mathrm{~min}^{-1}$ flow rate of the sample gas, $40 \mu \mathrm{L} \mathrm{min}^{-1}$ PFBHA derivatisation solution $\left(5.0 \times 10^{-4} \mathrm{~mol} \mathrm{~L}^{-1}\right)$ flow and both dicarbonyls at a mixing ratio of $50 \mathrm{ppbV}$, which implies a higher reaction temperature is favourable. The micro-reactor can be heated directly to an elevated temperature due to its planar shape, by a hot plate, unlike more commonly used bubblers and impingers that are often used for stripping and derivitising reactions. Temperature affects the derivatisation reaction in 

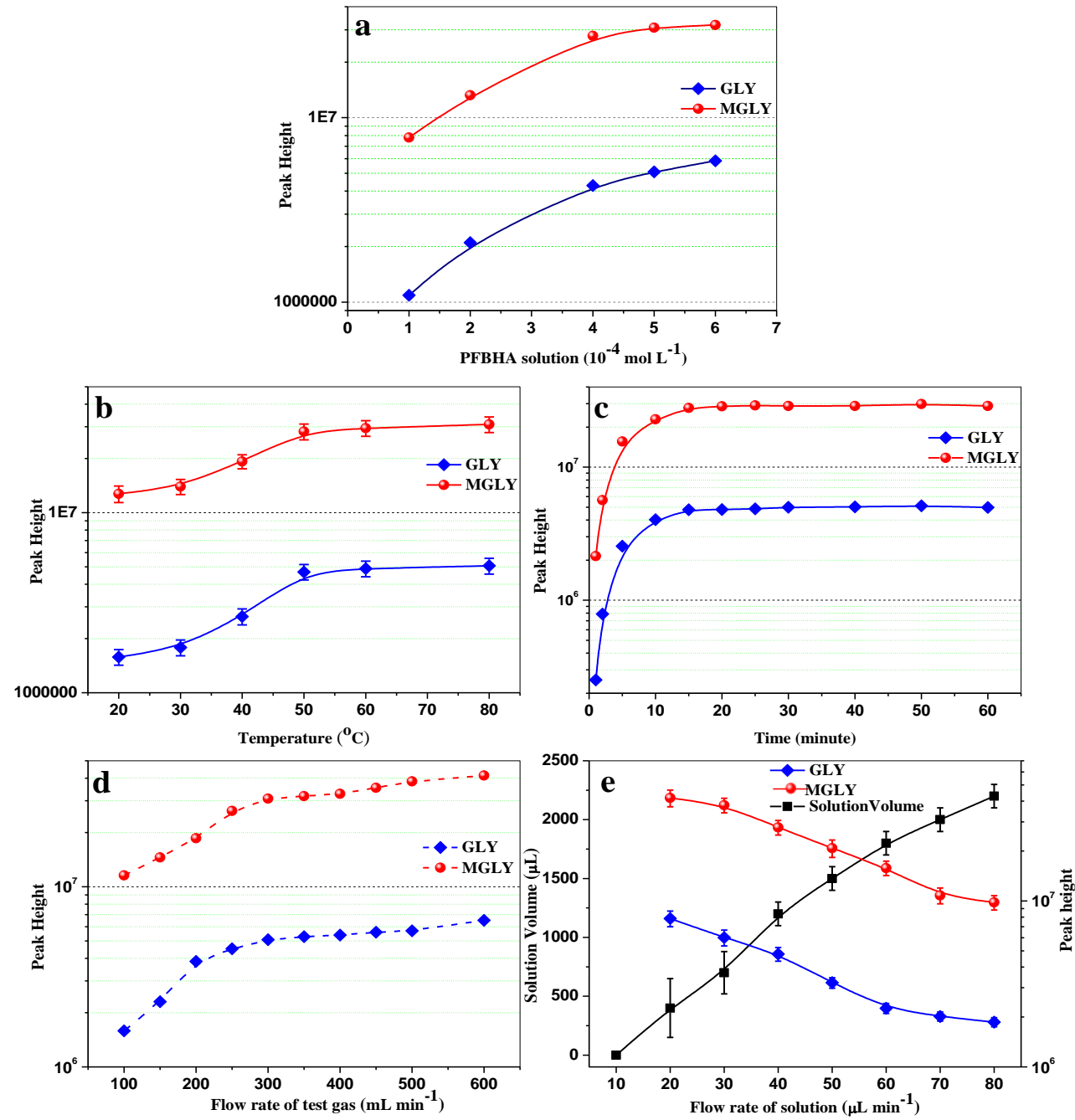

Fig. 3. Influences of PFBHA concentration (a), temperature of micro-reactor (b), reaction time (c), flow rates of GLY and MGLY gas (d) and flow rate of derivatisation solution (e) on the peak heights of derivatives in GC-MS chromatograms. Error bars indicate the relative standard deviations of GC-MS measurements. See text for details of the other experimental conditions.

the microchannel via three processes: (1) the reaction rate between $\alpha$-dicarbonyl and PFBHA (Destaillats and Charles, 2002), (2) the PFBHA and $\alpha$-dicarbonyl partitioning between the gas and the liquid phases, (3) the partitioning of the PFBHA- $\alpha$-dicarbonyl derivatives between the liquid and the gas phases. It should be noted that too high a temperature leads to rapid solvent evaporation. The optimal reaction temperature in this study was chosen as $60^{\circ} \mathrm{C}$.

A storage period from one hour to several days was found to increase PFBHA derivative yields in previous studies (Ho and $\mathrm{Yu}, 2002$; Temime et al., 2007). In this study, the residence time of the carbonyls in the micro-channel is a few minutes at most. The derivatisation reaction begins instantaneously but is not necessarily completed within the microchannel and a solution storage time of ten minutes was still needed to allow the derivatisation reaction to complete. In this study, the peak heights of derivatives in the GC-MS chromatogram reached their maximum and remained relatively constant after around fifteen minutes (Fig. 3c). Thus all samples in this study were injected into the GC-MS after a storage period of at least $15 \mathrm{~min}$, i.e., once the derivatisation reaction had reached its end point. Longer storage times were found to have no significant effect on the GC-MS signal responses of GLY and MGLY drivatives even after $24 \mathrm{~h}$ storage. Since the PFBHA derivatisation process is effectively a nucleophilic addition reaction the simple steric structures and electron configurations of GLY and MGLY are favourable to the faster reaction rate (Temime et al., 2007; Reisen et al., 2003). The derivatisation process occurring in the microreactor is considered sufficiently fast that it can be used for near real-time measurement of $\alpha$-dicarbonyls with the microreactor effluent passed directly for in situ GC-MS analysis. 
Such an approach circumvents the problems associated with slow storage reactions and long-term degradation of derivative samples in solution.

\subsection{Optimisation of flows of gas and solution}

A key function of the micro-reactor is to efficiently mix the gas flow containing the target $\alpha$-dicarbonyls with the PFBHA solution. If the flow rate of the test gas is too high, the $\alpha$ dicarbonyls do not have chance to dissolve in the wall liquid film and react causing the derivatisation efficiency to decrease. The overall derivatisation efficiency was influenced strongly by the mixing at the channel junction and downstream in the capillary region.

Flow rates of the sample gas were varied from 100 to $600 \mathrm{~mL} \mathrm{~min}^{-1}$ and evaluated for peak height with the derivatisation solution $\left(5.0 \times 10^{-4} \mathrm{~mol} \mathrm{~L}^{-1}\right)$ flow set at $40 \mu \mathrm{L} \mathrm{min}^{-1}$ and the temperature of micro-reactor at $60^{\circ} \mathrm{C}$ for both $\alpha$-dicarbonyls at a mixing ratio of $50 \mathrm{ppbV}$ (Fig. 3). At lower flow rates (between 0 and $300 \mathrm{~mL}^{-1}$ ) a stoichiometric increase in peak area is seen as the gas flow rate increases. Above this point, peak areas then increase more slowly. It should be noted that a too high gas flow rate can lead to excessive rapid solvent evaporation. In this study, the optimal flow of $\alpha$-dicarbonyl in gas was chosen as $200 \mathrm{~mL} \mathrm{~min}^{-1}$ to avoid the dilution effect to target compounds in EUPHORE chamber due to high gas flow rate. In Fig. 3e, five flow rates of derivatisation solution $\left(5.0 \times 10^{-4} \mathrm{~mol} \mathrm{~L}^{-1}\right)$, varying from 20 to $120 \mu \mathrm{L} \mathrm{min}^{-1}$ were assessed with the test gas flow of $200 \mathrm{~mL} \mathrm{~min}^{-1}$ and all other conditions the same as the experiment in Fig. 3d. Peak heights diminish as the liquid flow rate increases - a value of around $30-40 \mu \mathrm{L} \mathrm{min}^{-1}$ gave good peak heights whilst retaining sufficient workable volumes of solution in the reactor for subsequent handling by GC-MS (Fig. 3e). The optimal flow rate of PFBHA derivatisation solutions was chosen as $40 \mu \mathrm{L} \mathrm{min}{ }^{-1}$ for both GLY and MGLY detection. Based on the above optimisation processes, the optimal conditions of the micro-reactor for measurement of $\alpha$ dicarbonyls are established and shown in Table 1.

\subsection{Method calibration}

The method calibration curves for the microfluidic derivatisation technique were established based on measuring a series of GLY and MGLY gas mixtures of different concentrations prepared in the EUPHORE chamber. The GLY and MGLY standard gas mixtures in the chamber were simultaneously determined by the FTIR and BBCEAS techniques, which helped construct the calibration curves for the microfluidic derivatisation technique.

In practice the FTIR and BBCEAS instruments showed good agreement for the measurement of GLY and MGLY when dicarbonyl mixing ratios were above $5 \mathrm{ppbV}$ and 2 ppbV, respectively (Fig. 1a), although there is evidence of divergence at the lowest mixing ratios. Two versions of the

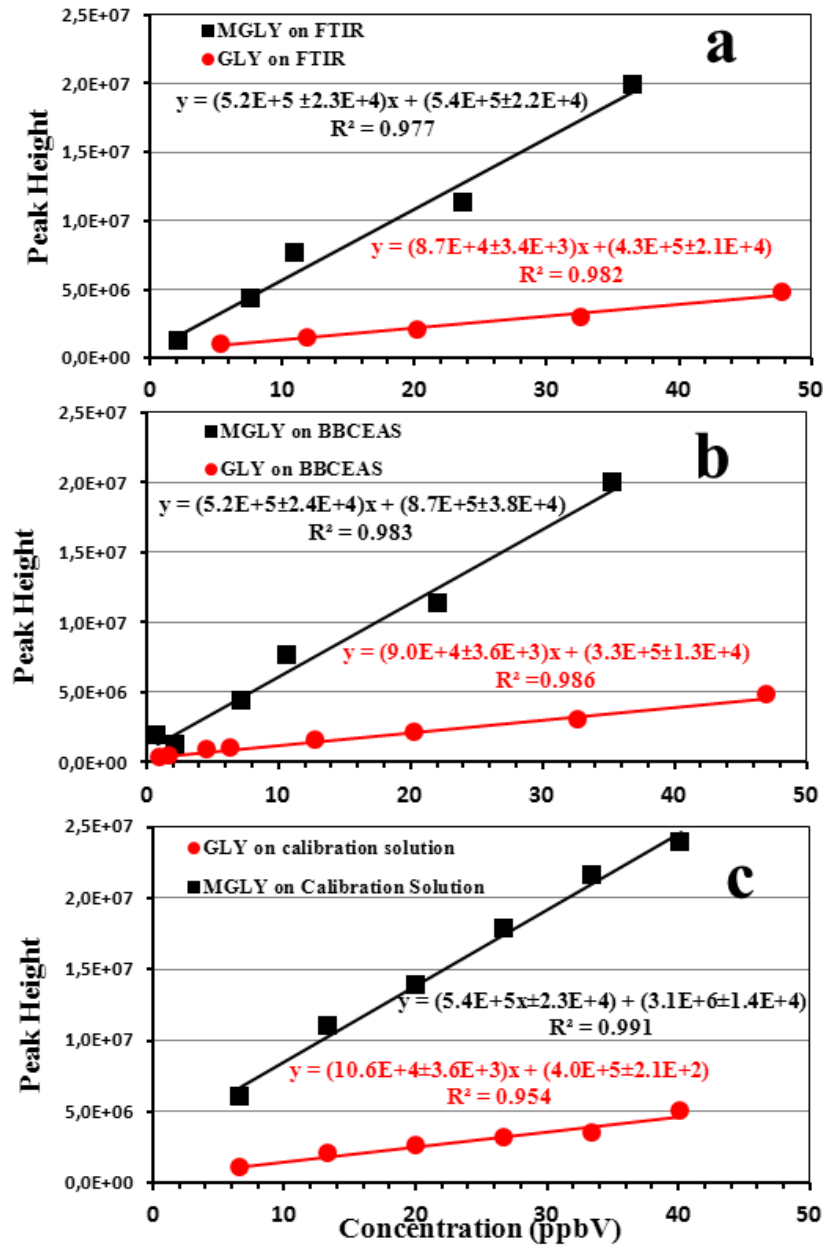

Fig. 4. Calibration curves of GLY and MGLY based on the absolute $\alpha$-dicarbonyl data provided by FTIR panel (a) and by BBCEAS panel (b) measured by the microfluidic derivatisation technique under the optimal conditions. Calibration curves of GLY and MGLY in panel (c) are established by the calibration solution of $\alpha$-dicarbonyl derivatives. The uncertainties of the slopes are less than $5 \%$.

calibration equations are obtained according to the two optical techniques employed as shown in Fig. 4. One version (Fig. 4a) is based on the absolute GLY and MGLY concentrations provided by FTIR and the other (Fig. 4b) references against the BBCEAS data. In Fig. 4a, GLY shows a good linearity from 5.4 to $48.2 \mathrm{ppbV}$ and MGLY from 2.1 to $37.1 \mathrm{ppbV}$ referenced to FTIR. In Fig. 4b, GLY shows a good linearity from 1.0 to $47.0 \mathrm{ppbV}$ and MGLY from 0.8 to $34.0 \mathrm{ppbV}$ by BBCEAS. The gradients and intercepts of the linear best fit lines for GLY in Fig. $3 a$ and $b$ are consistent with each other within the measurement errors of the FTIR and BBCEAS techniques (likewise for MGLY). Therefore, it can be concluded that there are no systematic differences among the calibrations equations established by the two independent reference techniques. Additionally, the correlation between FTIR and BBCEAS in GLY and MGLY 
determinations shown in Fig. $1 \mathrm{~b}$ and $\mathrm{c}$ also confirms the good agreement with the two reference techniques.

Another calibration method, based on measurements on standard solutions $\alpha$-dicarbonyl-PFBHA derivatives at different concentrations, is shown in Fig. 4c. The concentrations of the $\alpha$-dicarbonyl derivative solutions have been transformed into the mixing ratios of gaseous $\alpha$-dicarbonyls assuming the derivatisation efficiencies were $100 \%$. The gradients of the calibration equations based on standard solutions are higher than those based on the BBCEAS data with $7.5 \%$ and $6.0 \%$ increases for GLY and MGLY, respectively, which may be caused by the lower reaction efficiencies for the derivatisation reaction occurring in the micro-reactor than those in standard calibration solution. The reaction efficiencies of the derivatisation reaction in the micro-reactor were calculated as $92 \%$ and $93 \%$ for GLY and MGLY, respectively, based on the calculation method described in our previous study (Pang and Lewis, 2012), and these values are consistent with the derivatisation efficiencies deduced as $(100 \%-7.5 \%)=92 \%$ for gas-phase GLY and $(100 \%-$ $6 \%)=93 \%$ for gas-phase MGLY from the lower standard gas-phase calibrations (Fig. 4b) than the standard solution calibrations (Fig. 4c).

There are no systemic differences among the calibration equations established by the standard calibration solutions and optical measurements, which implies the calibration solution method is feasible for gaseous GLY and MGLY determinations. Thus the calibration equations based on liquid standard solution were chosen to quantify $\alpha$-dicarbonyl concentrations subsequently among the above three calibration curves. In this study, the definition of method detection limit (MDL) is the lowest concentration of analyte that gives a signal which is statistically significantly different from the blank signal. The MDL is calculated by applying the calibration fit coefficients to a peak height equal to the mean height from the blanks plus 3 times the standard deviation of blank values. The MDLs for the microfluidic method are estimated to be $76 \mathrm{pptV}$ for GLY and $185 \mathrm{pptV}$ for MGLY. The comparisons between the microfluidic PFBHA derivatisation technique and other previously reported methods for measuring GLY and MGLY, in terms of detection limit and linear range are listed in Table 2. The detection limit of this technique for GLY measurement is lower than those of FTIR, SPME, IBBCEAS (Washenfelder et al., 2008) and DOAS (Volkamer et al., 2005a) and is higher that of PFBHA mist chamber technique. But it should be noted that the other methods have shorter acquisition times than the microfluidic derivatisation technique and the sampling gas volume $(250-300 \mathrm{~L})$ collected from mist chambers is much higher than in this study (6L). For MGLY measurement the microfluidic technique is more sensitive than most spectrometric methods and PTR-MS (Washenfelder et al., 2008; Thalman and Volkamer, 2010; Volkamer et al., 2005a) but less than other PFBHA derivatisation methods (Spaulding et al., 1999, 2002) due to the significantly lower sample volumes. For time resolution, the microfluidic derivatisation technique is slower than all on-line spectrometric techniques since its sampling and measuring processes are separated. The instruments for online measurement can complete sampling and measurement simultaneously in a short time varying from $20 \mathrm{~s}$ (BBCEAS) up to $15 \mathrm{~min}$, whilst the microfluidic technique spends around $30 \mathrm{~min}$ to collect sample and $30 \mathrm{~min}$ to measure the sample by GC-MS. However, the microfluidic technique is considerably more rapid and more automated when compared with other offline chemical derivatisation techniques; these require 2-3 h sampling time and manual offline sampling treatments (Zhou and Mopper, 1990; Spaulding et al., 1999). The microfluidic method has a longer sampling time (30 min) than that of the SPME derivatisation technique (5 min) used in this study. It should be noted that the microfluidic method benefits from simplicity and cost, and can be available to most common laboratories with GC-MS or GC-FID. The automation potential, short sampling time and simple sampling treatment compared with other offline methods are significant advantages, making it potentially suitable for field measurements and long-term observations on GLY and MGLY in ambient air but clearly not for mobile applications such as aircraft measurements.

The average accuracies for GLY and MGLY are $6.6 \%$ and $7.5 \%$ which were calculated as the average relative error to the absolute concentrations provided by FTIR and BBCEAS, respectively. The precisions for GLY and MGLY are $11.2 \%$ and $11.4 \%$ respectively, which are the average Relative Standard Derivation (RSD) for the five parallel samples and five parallel injections. The detailed values for each measurement are shown in Table 3.

\subsection{Application and direct comparisons with other techniques}

To test the performance of the microfluidic derivatisation technique for time resolved measurements, the technique was employed to determine gaseous GLY and MGLY during an experiment in the EUPHORE chamber to study the photo-oxidation of isoprene under relatively low $\mathrm{NO}_{\mathrm{x}}$ $\left(\mathrm{NO}<2 \mathrm{ppbV}, \mathrm{NO}_{2} 13-21 \mathrm{ppbV}\right)$ conditions. The microfluidic derivatisation technique was compared with FTIR, BBCEAS, PTR-ToF-MS and SPME derivatisation techniques as well as the simulation by a chamber specific box model containing the complete MCMv3.2 isoprene scheme on the temporal evolution of the $\alpha$-dicarbonyl products during the experiment. Mixing ratios of GLY and MGLY were output from the model every $5 \mathrm{~min}$. The comparisons of the five analytical techniques and the MCM box model simulation are shown in Fig. 5. In the figure, each data point obtained by the microfluidic and SPME techniques show the average concentration during $30 \mathrm{~min}$ sampling period of each sample and the data point given by BBCEAS technique indicates the average concentration during $20 \mathrm{~s}$ period of each sample collection. Both FTIR and PTR-MS data points 
Table 3. Accuracy and precision of the microfluidic derivatisation technique

\begin{tabular}{|c|c|c|c|c|c|c|c|c|c|c|c|}
\hline \multicolumn{2}{|c|}{$\begin{array}{l}\text { Absolute mixing } \\
\text { ratio }(\mathrm{ppbV})^{\mathrm{a}}\end{array}$} & \multicolumn{2}{|c|}{$\begin{array}{l}\text { Observed mixing } \\
\text { ratio }(\mathrm{ppbV})^{\mathrm{b}}\end{array}$} & \multicolumn{2}{|c|}{$\begin{array}{c}\text { Accuracy } \\
(\%)^{\mathrm{c}}\end{array}$} & \multicolumn{2}{|c|}{$\begin{array}{l}\text { Precision of } \\
\text { reactor }(\%)^{d}\end{array}$} & \multicolumn{2}{|c|}{$\begin{array}{l}\text { Precision of } \\
\text { GC-MS }(\%)^{\mathrm{e}}\end{array}$} & \multicolumn{2}{|c|}{$\begin{array}{c}\text { Total precision } \\
\qquad \%)^{\mathrm{f}}\end{array}$} \\
\hline GLY & MGLY & GLY & MGLY & GLY & MGLY & GLY & MGLY & GLY & MGLY & GLY & MGLY \\
\hline 40.2 & 121.3 & 37.5 & 115.6 & 6.7 & 7.7 & 7.6 & 7.4 & 3.5 & 3.8 & 11 & 11.2 \\
\hline 29.5 & 85.0 & 28.3 & 81.5 & 6.3 & 7.1 & 7.5 & 6.9 & 3.4 & 3.9 & 10.9 & 11.1 \\
\hline 19.4 & 56.3 & 18.1 & 53.6 & 6.7 & 7.8 & 6.5 & 6.8 & 5.9 & 5.1 & 11.7 & 11.9 \\
\hline
\end{tabular}

${ }^{\mathrm{a}}$ Absolute concentrations of GLY and MGLY were given by BBCEAS. ${ }^{\mathrm{b}}$ Observed concentrations were measured by microfluidic derivatisation technique under optimal experimental conditions. ${ }^{c}$ Accuracy of the method was calculated by [(observed concentration-absolute concentration) /absolute concentration] $\times 100 \%$. ${ }^{\mathrm{d}}$ RSD of replicate analyses $(n=5)$ of standard $\alpha$-dicarbonyl gases. ${ }^{\mathrm{e}}$ RSD of repeated measurements $(n=5)$ under the same GC-MS instrumental conditions. Relative Standard Deviation (RSD) $=[($ standard deviation of observed concentration) $/($ average of observed concentration) $] \times 100 \%{ }^{\mathrm{f}}$ sum of precision of reactor and GC-MS
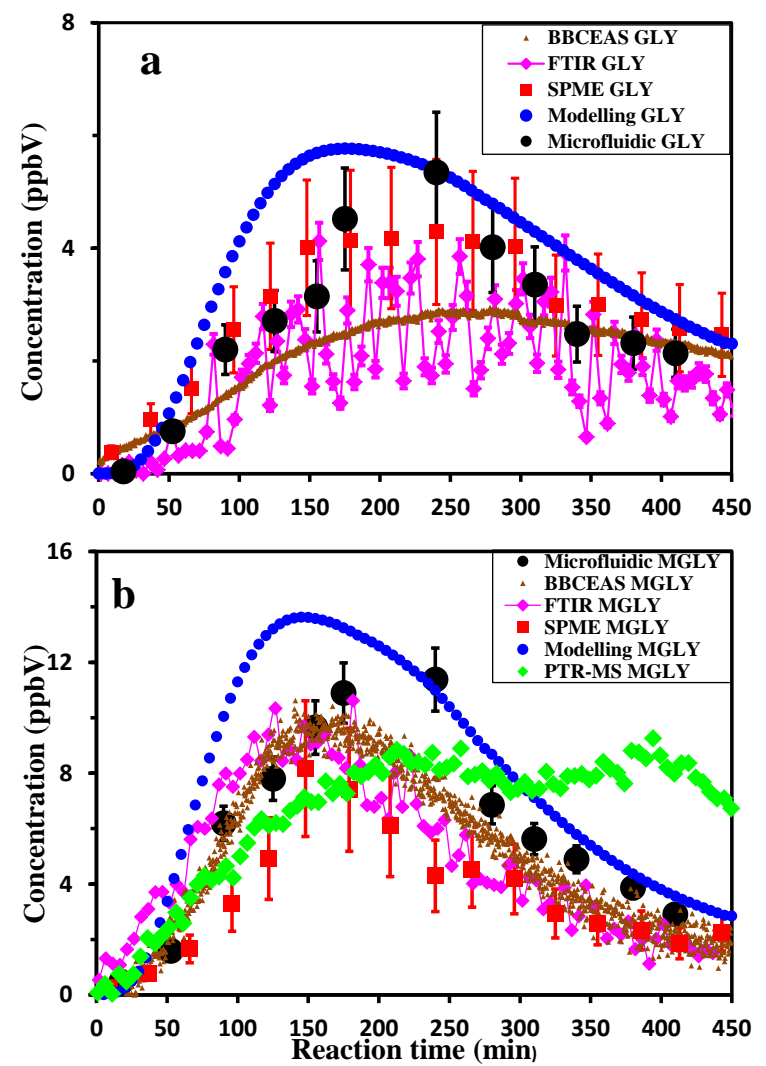

Fig. 5. Comparison of the microfluidic derivatisation technique, FTIR, BBCEAS, PTR-ToF-MS, SPME derivatisation technique and a MCM (v3.2) box model simulation on gaseous GLY and MGLY measurements during isoprene photo-oxidation under low NO condition in EUPHORE chamber. (a): Comparison of GLY measurement. (b): Comparison of MGLY measurement. Error bars show uncertainties of measurements: $6.7 \%$ for GLY and $8 \%$ for MGLY by microfluidic derivatisation and $30 \%$ for both $\alpha$-dicarbonyls by SPME.

imply the mean concentrations integrated from 5 min sampling time. The results of FTIR, BBCEAS, PTR-ToF-MS and the modelling simulation were averaged in accordance with the sampling time interval of microfluidic derivatisation samples. Therefore, the pairwise correlations were estimated based on the results on the same sampling period of the measurement techniques and the modelling simulation. The correlation results are shown in Table 4 including the correlation coefficients $\left(r^{2}\right)$, gradients and intercepts of the linear equations among all methods.

The comparison of GLY measurements by the various techniques and the MCM model is shown in Fig. 5a. Overall the microfluidic derivatisation technique showed a similar GLY evolution to those obtained from other methods. Better correlation was found between the microfluidic technique and SPME techniques with the $r^{2}$ and gradient values of 0.91 and $0.93(n=11)$ (Table 4), which implies the two techniques based on PFBHA chemical derivatisation have similar performances for GLY measurement. Good correlation coefficients were observed between the microfluidic derivatisation technique and FTIR, BBCEAS, MCM modelling simulation for GLY. The evolutions of GLY in Fig. 5a show the microfluidic method has two outliers compared with the other methods. The outlier at 240 min may be a result of more solvent evaporating from the solution vial than other samples (faulty cap for example), which gave higher concentrations of both GLY and MGLY. The explanation for those outliers after 175 min may be the memory effect from high GLY concentrations at 175 and $240 \mathrm{~min}$. To avoid the above potential problems an internal standard - a carbonyl that does not occur in the atmosphere could be added into the solution. An isotopically labelled version of the target carbonyls would also be an option as an internal standard. Heating the gas lines tubing before sampling may also reduce the GLY and MGLY absorption on tubing membrane. FTIR data points show more scatter than those of other techniques in Fig. 5a, which may be caused by FTIR unstable performance close to $2.5 \mathrm{ppbV}$ the FTIR detection limit.

The MGLY measurements by the various methods are shown in Fig. 5b. With the exception of the aforementioned outlier point at $240 \mathrm{~min}$, the microfluidic derivatisation technique shows a good agreement with BBCEAS and MCM modelling simulation on MGLY evolution with good correlation coefficients and gradients (Table 4). Those good 
Table 4. Correlation coefficients $\left(r^{2}\right)$, slopes (S) and intercepts (I) of the linear equations among the microfluidic derivatisation technique and other techniques in the measurement of $\alpha$-dicarbonyls during isoprene photooxidation in EUPHORE chamber based on the number $(n){ }^{\text {a }}$ of experimental data points. Correlations have been performed by averaging the data from the faster instrument over the acquisition time windows of the slower instrument.

\begin{tabular}{|c|c|c|c|c|c|c|c|c|c|c|c|}
\hline \multirow[b]{2}{*}{$Y \downarrow X \rightarrow$} & \multicolumn{5}{|c|}{ GLY } & \multicolumn{6}{|c|}{ MGLY } \\
\hline & Microfluidic $^{c}$ & BBCEAS & FTIR & SPME & Model & Microfluidic & BBCEAS & FTIR & SPME & Model & PTR-MS \\
\hline Microfluidic $^{\mathrm{b}}$ & 1 & & & & & 1 & & & & & \\
\hline BBCEAS & $\begin{array}{l}0.82\left(r^{2}\right) \\
0.56(\mathrm{~S}) \\
0.69(\mathrm{I})^{\mathrm{d}}\end{array}$ & 1 & & & & $\begin{array}{l}0.93 \\
0.93,-0.22\end{array}$ & 1 & & & & \\
\hline FTIR & $\begin{array}{l}0.81, \\
0.63,0.26\end{array}$ & $\begin{array}{l}0.65, \\
1.04,-0.29\end{array}$ & 1 & & & $\begin{array}{l}0.88, \\
0.83,0.24\end{array}$ & $\begin{array}{l}0.91, \\
0.99,0.08\end{array}$ & 1 & & & \\
\hline SPME & $\begin{array}{l}0.91 \\
0.83,0.74\end{array}$ & $\begin{array}{l}0.83 \\
1.35,0.11\end{array}$ & $\begin{array}{l}0.79 \\
1.06,1.17\end{array}$ & 1 & & $\begin{array}{l}0.93 \\
0.71,-0.11\end{array}$ & $\begin{array}{l}0.88 \\
0.67,0.37\end{array}$ & $\begin{array}{l}0.64, \\
0.63,0.62\end{array}$ & 1 & & \\
\hline Model & $\begin{array}{l}0.81, \\
1.07,0.85\end{array}$ & $\begin{array}{l}0.70 \\
1.72,0.14\end{array}$ & $\begin{array}{l}0.55 \\
1.19,1.2\end{array}$ & $\begin{array}{l}0.92, \\
1.46,-0.60\end{array}$ & 1 & $\begin{array}{l}0.86 \\
1.15,0.94\end{array}$ & $\begin{array}{l}0.96 \\
1.36,0.49\end{array}$ & $\begin{array}{l}0.83 \\
1.40,0.83\end{array}$ & $\begin{array}{l}0.83 \\
1.81,1.00\end{array}$ & 1 & \\
\hline PTR-MS & NA & NA & NA & NA & NA & $\begin{array}{l}0.39 \\
0.46,3.8\end{array}$ & $\begin{array}{l}0.21, \\
0.37,-4.69\end{array}$ & $\begin{array}{l}0.03 \\
0.15,5.74\end{array}$ & $\begin{array}{l}0.29 \\
0.61,4.2\end{array}$ & $\begin{array}{l}0.22 \\
0.28,4.4\end{array}$ & 1 \\
\hline
\end{tabular}

correlations imply microfluidic performance for MGLY is credible and broadly equivalent to other online techniques. The gradients less than 1 in the linear equations between microfluidic technique and other method imply microfluidic derivatisation measurements in MGLY are higher than other techniques in this experiment. There is one significant outlier at 240 min that shows MGLY concentration is still increasing measured by microfluidic method whilst the data from other methods are decreasing. This outlier may be ascribed to the higher enrichment ratio for this sample since more solvent evaporated from the derivative solution due to the longer sampling time (60min) of this sample compared with that of other sample (30 min). PTR-ToF-MS's results agree reasonably well with those obtained by other techniques during the initial $200 \mathrm{~min}$ of the comparison study, following which a divergence from the data recorded by other techniques is observed (Fig. 5a). After that time the MGLY signal of PTR-ToF-MS does not decay (whereas all other techniques do), but remains stable and constant for the remainder of the experiment. An explanation for this phenomenon may be that the isobaric background signal from the protonated water cluster $\left(\mathrm{H}_{2} \mathrm{O}\right)_{4} \cdot \mathrm{H}^{+}$increased with the increasing sample humidity and caused the interfering and overlapping signal on the $m / z 73$ mass channel, which is identical to $m / z$ of MGLY $\cdot \mathrm{H}^{+}$at the mass resolution of the instrument.

A comparison of the model vs. measurements in general shows that the model is over-predicting the absolute concentrations of GLY and MGLY but is doing a reasonable job of predicting the temporal profiles of both dicarbonyls throughout the experiment, giving us some confidence in the model chemistry (see Table 4). A more in-depth modelling study comparing the MCMv3.2 isoprene chemistry against a range of measured products (including other first/second/third generation oxygenates such as methacrolein, methyl vinyl ketone, hydroxyl acetone, glycolaldehyde), over a range of
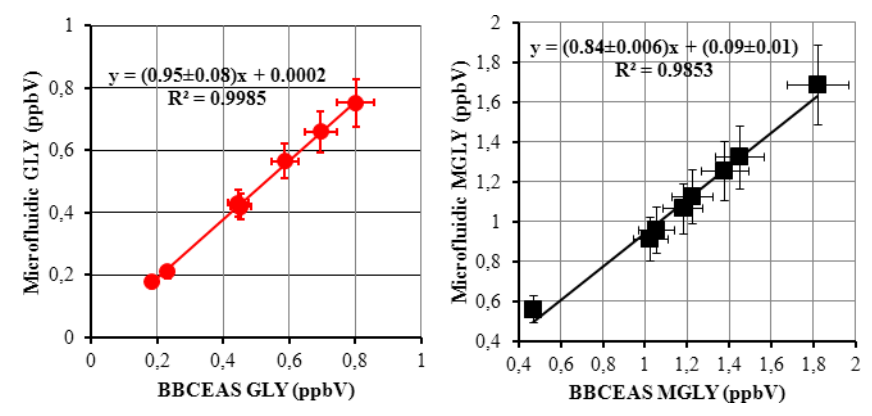

Fig. 6. Intercomparison of microfluidic derivatisation technique and BBCEAS for GLY and MGLY near detection limits. (a): linear relationship for GLY measurement (error bars for two techniques indicate the uncertainties); (b): linear relationship for MGLY measurement. The uncertainties of the slopes are less than $7 \%$.

atmospheric conditions, will be presented in a separate paper.

To further test the feasibility of the microfluidic derivatisation technique for measurements of GLY and MGLY close to the detection limit, a comparison was conducted between the microfluidic technique and BBCEAS at low GLY (0.1$0.8 \mathrm{ppbv}$ ) and MGLY (0.5-1.7 ppbv) concentrations (Fig. 6). Those samples were collected from the beginning or ends of some experiments when GLY and MGLY concentrations were low in the chamber. Good agreements are observed between the microfluidic derivatisation technique and BBCEAS in the measurements of GLY and MGLY at the lowest concentrations.

The similar performance of the microfluidic derivatisation technique with other online instrumental techniques and SPME derivatisation in EUPHORE chamber demonstrates the sensitivity and time resolution of microfluidic derivatisation technique is adequate for field GLY and MGLY measurements. Some further refinements of the microfluidic 
technique will be conducted to allow detection of ambient $\alpha$-dicarbonyl compounds principally through improvements in the ruggedness and automation of the micro-reactor and the inclusion of an internal standard.

It is appreciated that the above results are based on a small amount of data from a few experiments but we believe that this study indicates that the microfluidic derivatisation technique is a valuable and feasible analytical method for GLY, MGLY and potentially other trace atmospheric carbonyl compounds in ambient air.

\section{Conclusions}

A new and near automated microfluidic "lab-on-a-chip" derivatisation technique has been developed for the sensitive measurement of gas-phase glyoxal (GLY) and methylglyoxal (MGLY), and has been tested in a series of simulation experiments in the EUPHORE outdoor atmospheric simulation chamber. The method is based on microfluidic derivatisation of carbonyls and pre-concentration of the reaction solution in a glass micro-reactor followed by GCMS analysis. The microchannels of the reactor enhance the phase contact area-to-volume ratio, and allow direct heating of the on-chip reaction. This combination of small volumes and higher temperatures creates a much faster and higher efficiency derivatisation between $\alpha$-dicarbonyls and PFBHA than can be achieved using other traditional derivatisation methods and without requiring any additional preparative steps. Effluent leaving the micro-reactor can be directly injected into a standard bench-top GC-MS without further preparation. The technique was employed to measure the $\alpha$-dicarbonyl products formed during a controlled isoprene photo-oxidation experiment in the EUPHORE outdoor smog chamber, and was compared with several alternative spectroscopic and mass spectrometric techniques. The consistency in temporal evolutions of GLY and MGLY between different experimental measurement techniques and a detailed box model simulation provides some initial evidence for the effectiveness of the technique in practical application. The consistent calibration equations established by the standard calibration solutions and FTIR and BBCEAS measurements implies the calibration solution method is feasible for field determinations. The microfluidic derivatisation technique provides significant opportunities to build on the specificity of derivatisation for a series of carbonyls but at a low cost, without manual preparative steps and allows for faster reaction times and on-line coupling with widely available GC-MS. Since the detection limits of microfluidic derivatisation technique to GLY (0.07 ppbV) and MGLY (0.18 ppbV) are lower than many reported tropospheric mixing ratios (1-2 ppbV) and much lower than levels seen in direct emissions such as in biogenic burning plumes, the microfluidic derivatisation technique could be employed for ambient GLY and MGLY measurements with time resolution of $30 \mathrm{~min}$ or less.
Acknowledgements. The EUPHORE experiments were carried out as part of the Natural Environment Research Council (NERC) funded "Pho-SOA" project (NE/H021108/1). X. Pang acknowledges support from the Chinese National Natural Science Foundation $(41175110,41322035)$ and the CAS/SAFEA International Partnership Program for Creative Research Teams. X. Pang, A. R. Rickard, T. J. Adams, S. M. Ball and M. J. S. Daniels acknowledge the National Centre for Atmospheric Science (NCAS) for support with travel, subsistence and instrument shipping expenses. T. J. Adams, M. J. S. Daniels and I. C. A. Goodall were supported on $\mathrm{PhD}$ studentships funded by NERC. The Instituto Universitario CEAM-UHM is partly supported by Generalitat Valenciana, and the projects GRACCIE (Consolider Ingenio 2010) and FEED-BACKS (Prometeo-Generalitat Valenciana). EUPHORE instrumentation is partly funded by the Spanish Ministry of Economy, through INNPLNATA project_PCT-440000-2010-003.

Edited by: A. Hofzumahaus

\section{References}

Blake, R. S., Monks, P. S., and Ellis, A. M.: Proton-Transfer Reaction Mass Spectrometry, Chemical Rev., 109, 861-896, 2009.

Bloss, C., Wagner, V., Bonzanini, A., Jenkin, M. E., Wirtz, K., Martin-Reviejo, M., and Pilling, M. J.: Evaluation of detailed aromatic mechanisms (MCMv3 and MCMv3.1) against environmental chamber data, Atmos. Chem. Phys., 5, 623-639, doi:10.5194/acp-5-623-2005, 2005.

Calvert, J. G., Atkinson, R., Kerr, J. A., Madronich, S., Moortgat, G. K., Wallington, T. J., and Yarwood, G.: The Mechanisms of Atmospheric Oxidation of the Alkenes, Oxford University Press, New York, 2000.

Calvert, J. G., Atkinson, R., Becker, K. H., Kamens, R. M., Seinfeld, J. H., Wallington, T. J., and Yarwood, G.: The Mechanisms of Atmospheric Oxidation of Aromatic Hydrocarbons, Oxford University Press, 2002.

Chan, A. W. H., Galloway, M. M., Kwan, A. J., Chhabra, P. S., Keutsch, F. N., Wennberg, P. O., Flagan, R. C., and Seinfeld, J. H.: Photooxidation of 2-Methyl-3-Buten-2-ol (MBO) as a Potential Source of Secondary Organic Aerosol, Environ. Sci. Technol., 43, 4647-4652, 2009.

Connelly, B. M., De Haan, D. O., and Tolbert, M. A.: Heterogeneous Glyoxal Oxidation: A Potential Source of Secondary Organic Aerosol, J. Phys. Chem. A, 116, 6180-6187, 2012.

De Haan, D. O., Corrigan, A. L., Tolbert, M. A., Jimenez, J. L., Wood, S. E., and Turley, J. J.: Secondary Organic Aerosol Formation by Self-Reactions of Methylglyoxal and Glyoxal in Evaporating Droplets, Environ. Sci. Technol., 43, 8184-8190, 2009.

Destaillats, H. and Charles, M. J.: Henry's Law Constants of Carbonyl-Pentafluorobenzyl Hydroxylamine (PFBHA) Derivatives in Aqueous Solution, J. Chem. Eng. Data, 47, 1481-1487, 2002.

Fu, T.-M., Jacob, D. J., Wittrock, F., Burrows, J. P., Vrekoussis, M., and Henze, D. K.: Global budgets of atmospheric glyoxal and methylglyoxal, and implications for formation of secondary organic aerosols, J. Geophys. Res., 113, D15303, doi:10.1029/2007JD009505, 2008.

Galloway, M. M., Huisman, A. J., Yee, L. D., Chan, A. W. H., Loza, C. L., Seinfeld, J. H., and Keutsch, F. N.: Yields of oxidized 
volatile organic compounds during the $\mathrm{OH}$ radical initiated oxidation of isoprene, methyl vinyl ketone, and methacrolein under high- $\mathrm{NO}_{\mathrm{x}}$ conditions, Atmos. Chem. Phys., 11, 10779-10790, doi:10.5194/acp-11-10779-2011, 2011.

Gomez Alvarez, E., Moreno, M. V., Gligorovski, S., Wortham, H., and Cases, M. V. R.: Characterisation and calibration of active sampling Solid Phase Microextraction applied to sensitive determination of gaseous carbonyls, Talanta, 88, 252-258, 2012.

Guenther, A., Hewitt, C. N., Erickson, D., Fall, R., Geron, C., Graedel, T., Harley, P., Klinger, L., Lerdau, M., McKay, W. A., Pierce, T., Scholes, B., Steinbrecher, R., Tallamraju, R., Taylor, J., and Zimmerman, P.: A global model of natural volatile organic compound emissions, J. Geophys. Res., 100, 8873-8892, 1995.

Gurnick, M., Chaiken, J., Thomas, B., and McDonald, J. D.: Vibrational and rotational spectroscopy of the first electronically allowed transition of alpha-dicarbonyls, J. Chem. Phys., 74, 99$105,1981$.

Hallquist, M., Wenger, J. C., Baltensperger, U., Rudich, Y., Simpson, D., Claeys, M., Dommen, J., Donahue, N. M., George, C., Goldstein, A. H., Hamilton, J. F., Herrmann, H., Hoffmann, T., Iinuma, Y., Jang, M., Jenkin, M. E., Jimenez, J. L., Kiendler-Scharr, A., Maenhaut, W., McFiggans, G., Mentel, Th. F., Monod, A., Prévôt, A. S. H., Seinfeld, J. H., Surratt, J. D., Szmigielski, R., and Wildt, J.: The formation, properties and impact of secondary organic aerosol: current and emerging issues, Atmos. Chem. Phys., 9, 5155-5236, doi:10.5194/acp-9-51552009, 2009.

Hamilton, J. F., Baeza-Romero, M. T., Finessi, E., Rickard, A. R., Healy, R. M., Peppe, S., Adams, T. J., Daniels, M J. S., Ball, S. M., Goodall, I. C. A., Monks, P. S., Borras, E., and Munoz, A.: Online and offline mass spectrometric study of the impact of oxidation and ageing on glyoxal chemistry and uptake onto ammonium sulphate aerosols, Faraday. Discuss., 165, 447-472, doi:10.1039/C3FD00051F, 2013.

Heland, J. R., Kleffmann, J. R., Kurtenbach, R., and Wiesen, P.: A New Instrument To Measure Gaseous Nitrous Acid (HONO) in the Atmosphere, Environ. Sci. Technol., 35, 3207-3212, 2001.

Henry, S. B., Kammrath, A., and Keutsch, F. N.: Quantification of gas-phase glyoxal and methylglyoxal via the LaserInduced Phosphorescence of (methyl)GLyOxal Spectrometry (LIPGLOS) Method, Atmos. Meas. Tech., 5, 181-192, doi:10.5194/amt-5-181-2012, 2012.

Ho, S. S. H. and Yu, J. Z.: Feasibility of Collection and Analysis of Airborne Carbonyls by On-Sorbent Derivatisation and Thermal Desorption, Anal. Chem., 74, 1232-1240, 2002.

Ho, S. S. H. and Yu, J. Z.: Determination of Airborne Carbonyls: Comparison of a Thermal Desorption/GC Method with the Standard DNPH/HPLC Method, Environ. Sci. Technol., 38, 862-870, 2004.

Hoffmann, T., Odum, J. R., Bowman, F., Collins, D., Klockow, D., Flagan, R. C., and Seinfeld, J. H.: Formation of Organic Aerosols from the Oxidation of Biogenic Hydrocarbons, J. Atmos. Chem., 26, 189-222, 1997.

Huisman, A. J., Hottle, J. R., Coens, K. L., DiGangi, J. P., Galloway, M. M., Kammrath, A., and Keutsch, F. N.: Laser-Induced Phosphorescence for the in Situ Detection of Glyoxal at Part per Trillion Mixing Ratios, Anal. Chem., 80, 5884-5891, 2008.

Huisman, A. J., Hottle, J. R., Galloway, M. M., DiGangi, J. P., Coens, K. L., Choi, W., Faloona, I. C., Gilman, J. B., Kuster, W. C., de Gouw, J., Bouvier-Brown, N. C., Goldstein, A. H., LaFranchi, B. W., Cohen, R. C., Wolfe, G. M., Thornton, J. A., Docherty, K. S., Farmer, D. K., Cubison, M. J., Jimenez, J. L., Mao, J., Brune, W. H., and Keutsch, F. N.: Photochemical modeling of glyoxal at a rural site: observations and analysis from BEARPEX 2007 , Atmos. Chem. Phys., 11, 8883-8897, doi:10.5194/acp-11-88832011, 2011.

Langridge, J. M., Ball, S. M., Shillings, A. J. L., and Jones, R. L.: A broadband absorption spectrometer using light emitting diodes for ultrasensitive, in situ trace gas detection, Rev. Sci. Instrum., 79, 123110-123110-123114, 2008.

Lee, Y.-N., Zhou, X., and Hallock, K.: Atmospheric carbonyl compounds at a rural southeastern United States site, J. Geophys. Res., 100, 25933-25944, 1995.

Lee, Y. N., Zhou, X., Kleinman, L. I., Nunnermacker, L. J., Springston, S. R., Daum, P. H., Newman, L., Keigley, W. G., Holdren, M. W., Spicer, C. W., Young, V., Fu, B., Parrish, D. D., Holloway, J., Williams, J., Roberts, J. M., Ryerson, T. B., and Fehsenfeld, F. C.: Atmospheric chemistry and distribution of formaldehyde and several multioxygenated carbonyl compounds during the 1995 Nashville Middle Tennessee Ozone Study, J. Geophys. Res.-Atmos., 103, 22449-22462, doi:10.1029/98jd01251, 1998.

Lerot, C., Stavrakou, T., De Smedt, I., Müller, J.-F., and Van Roozendael, M.: Glyoxal vertical columns from GOME-2 backscattered light measurements and comparisons with a global model, Atmos. Chem. Phys., 10, 12059-12072, doi:10.5194/acp10-12059-2010, 2010.

Loeffler, K. W., Koehler, C. A., Paul, N. M., and De Haan, D. O.: Oligomer Formation in Evaporating Aqueous Glyoxal and Methyl Glyoxal Solutions, Environ. Sci. Technol., 40, 63186323, 2006.

MacDonald, S. M., Oetjen, H., Mahajan, A. S., Whalley, L. K., Edwards, P. M., Heard, D. E., Jones, C. E., and Plane, J. M. C.: DOAS measurements of formaldehyde and glyoxal above a south-east Asian tropical rainforest, Atmos. Chem. Phys., 12, 5949-5962, doi:10.5194/acp-12-5949-2012, 2012.

Magneron, I., Mellouki, A., Le Bras, G., Moortgat, G. K., Horowitz, A., and Wirtz, K.: Photolysis and OH-Initiated Oxidation of Glycolaldehyde under Atmospheric Conditions, J. Phys. Chem. A, 109, 4552-4561, 2005.

Mauderly, J. L. and Chow, J. C.: Health Effects of Organic Aerosols, Inhalation Toxicology, 20, 257-288, doi:10.1080/08958370701866008, 2008.

Munger, J. W., Jacob, D. J., Daube, B. C., Horowitz, L. W., Keene, W. C., and Heikes, B. G.: Formaldehyde, glyoxal, and methylglyoxal in air and cloudwater at a rural mountain site in central Virginia, J. Geophys. Res., 100, 9325-9333, 1995.

Muñoz, A., Vera, T., Sidebottom, H., Mellouki, A., Borras, E., Rodenas, M., Clemente, E., and Vazquez, M.: Studies on the Atmospheric Degradation of Chlorpyrifos-Methyl, Environ. Sci. Technol., 45, 1880-1886, 2011.

Myriokefalitakis, S., Vrekoussis, M., Tsigaridis, K., Wittrock, F., Richter, A., Brühl, C., Volkamer, R., Burrows, J. P., and Kanakidou, M.: The influence of natural and anthropogenic secondary sources on the glyoxal global distribution, Atmos. Chem. Phys., 8, 4965-4981, doi:10.5194/acp-8-4965-2008, 2008. 
Pang, X. and Lewis, A. C.: Carbonyl compounds in gas and particle phases of mainstream cigarette smoke, Sci. Total Environ., 409, 5000-5009, 2011.

Pang, X. and Lewis, A. C.: A microfluidic lab-on-chip derivatisation technique for the measurement of gas phase formaldehyde, Analytical Methods, 4, 2013-2020, 2012.

Pang, X., Lewis, A. C., and Hamilton, J. F.: Determination of airborne carbonyls via pentafluorophenylhydrazine derivatisation by GC-MS and its comparison with HPLC method, Talanta, 85, 406-414, 2011

Pang, X., Lewis, A. C., and Ródenas-García, M.: Microfluidic labon-a-chip derivatisation for gaseous carbonyl analysis, J. Chromatography A, 1296, 93-103, 2013.

Profeta, L. T. M., Sams, R. L., Johnson, T. J., and Williams, S. D.: Quantitative Infrared Intensity Studies of Vapor-Phase Glyoxal, Methylglyoxal, and 2,3-Butanedione (Diacetyl) with Vibrational Assignments, J. Phys. Chem. A, 115, 9886-9900, 2012.

Reisen, F., Aschmann, S. M., Atkinson, R., and Arey, J.: Hydroxyaldehyde Products from Hydroxyl Radical Reactions of Z-3Hexen-1-ol and 2-Methyl-3-buten-2-ol Quantified by SPME and API-MS, Environ. Sci. Technol., 37, 4664-4671, 2003.

Rickard, A. R., Wyche, K. P., Metzger, A., Monks, P. S., Ellis, A. M., Dommen, J., Baltensperger, U., Jenkin, M. E., and Pilling, M. J.: Gas phase precursors to anthropogenic secondary organic aerosol: Using the Master Chemical Mechanism to probe detailed observations of 1,3,5-trimethylbenzene photo-oxidation, Atmos. Environ., 44, 5423-5433, 2010.

Ródenas, M.: Improvements in spectroscopy data processing: faster production and better reliability of Lab data, INTROP Report, 27770, available at: http://www.ceam.es/GVAceam/archivos/ MRodenasINTROPReport.pdf (last access: January 2014), 2008.

Saunders, S. M., Jenkin, M. E., Derwent, R. G., and Pilling, M. J.: Protocol for the development of the Master Chemical Mechanism, MCM v3 (Part A): tropospheric degradation of nonaromatic volatile organic compounds, Atmos. Chem. Phys., 3, 161-180, doi:10.5194/acp-3-161-2003, 2003.

Sinreich, R., Coburn, S., Dix, B., and Volkamer, R.: Ship-based detection of glyoxal over the remote tropical Pacific Ocean, Atmos. Chem. Phys., 10, 11359-11371, doi:10.5194/acp-1011359-2010, 2010.

Solomon, S., Qin, D., Manning, M., Chen, Z., Marquis, M., Averyt, K. B., Tignor, M., and Miller, H. L. (Eds.): The Physical Science Basis Contribution of Working Group I to the Fourth Assessment Report of the Intergovernmental Panel on Climate Change, Climate Change 2007, Cambridge University Press, Cambridge, United Kingdom and New York, NY, USA, 2007.

Spaulding, R. S., Frazey, P. A., Rao, X., and Charles, M. J.: Measurement of Hydroxy Carbonyls and Other Carbonyls in Ambient Air Using Pentafluorobenzyl Alcohol as a Chemical Ionization Reagent, Anal. Chem., 71, 3420-3427, 1999.

Spaulding, R. S., Talbot, R. W., and Charles, M. J.: Optimisation of a Mist Chamber (Cofer Scrubber) for Sampling Water-Soluble Organics in Air, Environ. Sci. Technol., 36, 1798-1808, 2002.

Spaulding, R. S., Schade, G. W., Goldstein, A. H., and Charles, M. J.: Characterization of secondary atmospheric photooxidation products: Evidence for biogenic and anthropogenic sources, J. Geophys. Res., 108, 4247, doi:10.1029/2002JD002478, 2003.

Talukdar, R. K., Zhu, L., Feierabend, K. J., and Burkholder, J. B.: Rate coefficients for the reaction of methylglyoxal
$\left(\mathrm{CH}_{3} \mathrm{COCHO}\right)$ with $\mathrm{OH}$ and $\mathrm{NO}_{3}$ and glyoxal $(\mathrm{HCO})_{2}$ with $\mathrm{NO}_{3}$, Atmos. Chem. Phys., 11, 10837-10851, doi:10.5194/acp11-10837-2011, 2011.

Temime, B., Healy, R. M., and Wenger, J. C.: A Denuder-Filter Sampling Technique for the Detection of Gas and Particle Phase Carbonyl Compounds, Environ. Sci. Technol., 41, 6514-6520, 2007.

Thalman, R. and Volkamer, R.: Inherent calibration of a blue LED-CE-DOAS instrument to measure iodine oxide, glyoxal, methyl glyoxal, nitrogen dioxide, water vapour and aerosol extinction in open cavity mode, Atmos. Meas. Tech., 3, 1797-1814, doi:10.5194/amt-3-1797-2010, 2010.

USA-EPA: Compendium method TO-11A.Determination of formaldehyde in ambient air using adsorbent cartridge followed by high performance liquid chromatography (HPLC) [active sampling methodology], 1999.

Volkamer, R., Molina, L. T., Molina, M. J., Shirley, T., and Brune, W. H.: DOAS measurement of glyoxal as an indicator for fast VOC chemistry in urban air, Geophys. Res. Lett., 32, L08806, doi:10.1029/2005GL022616, 2005a.

Volkamer, R., Spietz, P., Burrows, J., and Platt, U: High-resolution absorption cross-section of glyoxal in the UV-vis and IR spectral ranges., J. Photochem. Photobiol. A: Chemistry, 172, 35-46, $2005 b$.

Volkamer, R., San Martini, F., Molina, L. T., Salcedo, D., Jimenez, J. L., and Molina, M. J.: A missing sink for gas-phase glyoxal in Mexico City: Formation of secondary organic aerosol, Geophys. Res. Lett., 34, L19807, doi:10.1029/2007GL030752, 2007.

Volkamer, R., Ziemann, P. J., and Molina, M. J.: Secondary Organic Aerosol Formation from Acetylene $\left(\mathrm{C}_{2} \mathrm{H}_{2}\right)$ : seed effect on SOA yields due to organic photochemistry in the aerosol aqueous phase, Atmos. Chem. Phys., 9, 1907-1928, doi:10.5194/acp9-1907-2009, 2009.

Vrekoussis, M., Wittrock, F., Richter, A., and Burrows, J. P.: Temporal and spatial variability of glyoxal as observed from space, Atmos. Chem. Phys., 9, 4485-4504, doi:10.5194/acp-9-44852009, 2009.

Vrekoussis, M., Wittrock, F., Richter, A., and Burrows, J. P.: GOME-2 observations of oxygenated VOCs: what can we learn from the ratio glyoxal to formaldehyde on a global scale?, Atmos. Chem. Phys., 10, 10145-10160, doi:10.5194/acp-1010145-2010, 2010.

Washenfelder, R. A., Langford, A. O., Fuchs, H., and Brown, S. S.: Measurement of glyoxal using an incoherent broadband cavity enhanced absorption spectrometer, Atmos. Chem. Phys., 8, 7779-7793, doi:10.5194/acp-8-7779-2008, 2008.

Washenfelder, R. A., Young, C. J., Brown, S. S., Angevine, W. M., Atlas, E. L., Blake, D. R., Bon, D. M., Cubison, M. J., de Gouw, J. A., Dusanter, S., Flynn, J., Gilman, J. B., Graus, M., Griffith, S., Grossberg, N., Hayes, P. L., Jimenez, J. L., Kuster, W. C., Lefer, B. L., Pollack, I. B., Ryerson, T. B., Stark, H., Stevens, P. S., and Trainer, M. K.: The glyoxal budget and its contribution to organic aerosol for Los Angeles, California, during CalNex 2010, J. Geophys. Res., 116, D00V02, doi:10.1029/2011JD016314, 2011.

Wittrock, F., Richter, A., Oetjen, H., Burrows, J. P., Kanakidou, M., Myriokefalitakis, S., Volkamer, R., Beirle, S., Platt, U., and Wagner, T.: Simultaneous global observations of glyoxal and 
formaldehyde from space, Geophys. Res. Lett., 33, L16804, doi:10.1029/2006GL026310, 2006.

Yu, J., Jeffries, H. E., and Le Lacheur, R. M.: Identifying Airborne Carbonyl Compounds in Isoprene Atmospheric Photooxidation Products by Their PFBHA Oximes Using Gas Chromatography/Ion Trap Mass Spectrometry, Environ. Sci. Technol., 29, 1923-1932, 1995.
Zhou, X. L. and Mopper, K.: Measurement of sub-parts-per-billion levels of carbonyl compounds in marine air by a simple cartridge trapping procedure followed by liquid chromatography, Environ. Sci. Technol., 24, 1482-1485, 1990. 